\title{
Electric field measurements at the plasma frequency around comet 67P by RPC-MIP on board Rosetta
}

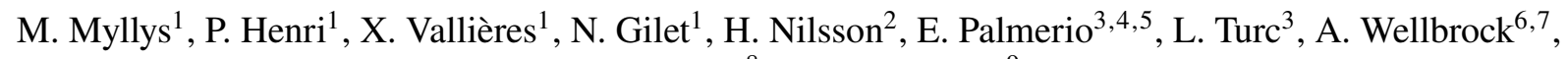 \\ R. Goldstein ${ }^{8}$, and O. Witasse ${ }^{9}$ \\ ${ }^{1}$ Laboratoire de Physique et Chimie de l'Environnement et de l'Espace (LPC2E), CNRS, Orléans, France \\ e-mail: myllysminna@gmail.com \\ ${ }^{2}$ Swedish Institute of Space Physics, PO Box 821, 98128 Kiruna, Sweden \\ ${ }^{3}$ Department of Physics, University of Helsinki, PO Box 64, 00014 Helsinki, Finland \\ ${ }^{4}$ Space Sciences Laboratory, University of California-Berkeley, Berkeley, CA 94720, USA \\ 5 CPAESS, University Corporation for Atmospheric Research, Boulder, CO 80301, USA \\ ${ }^{6}$ Mullard Space Science Laboratory, University College London, Holmbury St. Mary, Dorking, Surrey RH5 6NT, UK \\ 7 The Centre for Planetary Sciences at University College London/Birkbeck, Gower Street, London WC1E 6BT, UK \\ ${ }^{8}$ Southwest Research Institute, PO Drawer 28510, San Antonio, TX 78228-0510, USA \\ 9 European Space Agency (ESA), European Space Research and Technology Centre (ESTEC), Keplerlaan 1, 2201 AZ Noordwijk, \\ The Netherlands
}

Received 4 September 2019 / Accepted 12 April 2021

\begin{abstract}
Context. The Mutual Impedance Probe (RPC-MIP) carried by the Rosetta spacecraft monitored both the plasma density and the electric field in the close environment of comet 67P/Churyumov-Gerasimenko (67P), as the instrument was operating alternatively in two main modes: active and passive. The active mode is used primarily to perform plasma density measurements, while the passive mode enables the instrument to work as a wave analyzer.

Aims. We are reporting electric field emissions at the plasma frequency near comet 67P observed by RPC-MIP passive mode. The electric field emissions are related to Langmuir waves within the cometary ionized environment. In addition, this study gives feedback on the density measurement capability of RPC-MIP in the presence of cold electrons.

Methods. We studied the occurrence rate of the electric field emissions as well as their dependence on solar wind structures like stream interaction regions (SIRs) and coronal mass ejections (CMEs).

Results. We are showing that strong electric field emissions at the plasma frequency near 67P were present sporadically throughout the period when Rosetta was escorting the comet, without being continuous, as the occurrence rate is reported to be of about $1 \%$ of all the measured RPC-MIP passive spectra showing strong electric field emissions. The Langmuir wave activity monitored by RPC-MIP showed measurable enhancements during SIR or CME interactions and near perihelion.

Conclusions. According to our results, Langmuir waves are a common feature at 67P during the passage of SIRs. Comparing the plasma frequency given by the RPC-MIP passive mode during Langmuir wave periods with the RPC-MIP active mode observations, we conclude that the measurement accuracy of RPC-MIP depends on the operational submode when the cold electron component dominates the electron density.
\end{abstract}

Key words. comets: general - methods: data analysis - waves

\section{Introduction}

The European Space Agency (ESA) Rosetta mission escorted a comet, 67P/Churyumov-Gerasimenko (67P), for the first time along its path around the Sun and monitored its plasma environment between years 2014 and 2016. The measurements taken by the Rosetta orbiter spacecraft form a unique data set of observations during different phases of cometary activity. Rosetta was carrying five instruments designed to study the near-comet plasma and electromagnetic field environment, the Rosetta Plasma Consortium (RPC; Carr et al. 2007). One of the RPC instruments, the Mutual Impedance Probe (RPC-MIP; Trotignon et al. 2007), had the primary task of estimating the near-comet plasma density. Similar mutual impedance experiments will be carried by future missions. The Hermean magnetosphere will be studied by the Active Measurement of Mercury's Plasma ( $\mathrm{AM}^{2} \mathrm{P}$; Trotignon et al. 2006) instrument as part of the
Plasma Wave Investigation (PWI; Kasaba et al. 2020) on board BepiColombo (Milillo et al. 2020). The Mutual Impedance Measurement (MIME) will be part of the Radio and Plasma Wave Investigation (RPWI) carried by the Jupiter ICy Moons Explorer (JUICE; Grasset et al. 2013) mission that will observe plasma properties in the Jovian system.

The Rosetta RPC-MIP instrument is a sensor that consists of two transmitting and two receiving electric antennas. The basic function of the instrument is to measure the transfer electric impedance between the transmitter and receiving dipoles. The instrument is operating in two main modes: active and passive. In active mode, an oscillating electric field is induced through the transmitters to cause oscillations in the surrounding plasma. In passive mode, the RPC-MIP transmitters are instead switched off and the receiving dipole is used as a passive electric field antenna. While in active mode, the instrument is measuring the response of the plasma to the perturbation 
caused by the instrument itself, with the aim of extracting useful plasma properties; in passive mode, the received signal is caused by natural plasma processes. The RPC-MIP is able to observe the electric field component parallel to its receiving antenna. The frequency range of operation of RPC-MIP is $7 \mathrm{kHZ}$ to a few $\mathrm{MHz}$. In typical cometary conditions encountered by Rosetta the waves that RPC-MIP is able to measure are Langmuir waves. Langmuir waves are electrostatic waves oscillating at frequencies close to the plasma frequency. They have been observed in many different plasma environments in the heliosphere; for example, in planetary foreshocks (e.g., Crawford et al. 1990; Scarf et al. 1971; Lacombe et al. 1985), magnetotails (e.g., Deng et al. 2004), and ionospheres (e.g., McFadden et al. 1986), as well as in the interplanetary medium near shocks (e.g., Thejappa \& MacDowall 2000; Pulupa et al. 2010) or after solar flares (Henri et al. 2009). In addition, Langmuir waves have been observed in cometary environments during the flybys of comet 1P/Halley and comet 21P/Giacobini-Zinner. The International Cometary Explorer measured Langmuir wave activity upstream of the cometary shock of comet 21P (Kennel et al. 1986), while Vega observed Langmuir waves near comet 1P (Grard et al. 1986).

Langmuir waves are often explained as being caused by a bump-on-tail instability when a beam of high-energy electrons is streaming through the plasma in the direction parallel to the ambient magnetic field. Such electron beams can form because of electron acceleration at shocks, like the shocks driven by interplanetary coronal mass ejections (CMEs; Treumann 2009), or in magnetic reconnection regions (Deng et al. 2004), or even because of local electron heating (Briand et al. 2007). As such, they are a useful tracer of various processes associated to energy transfer such as local electron heating or parallel acceleration.

The goals of this paper are (i) to report the electric field observations of the RPC-MIP instrument as the Rosetta orbiter was monitoring the cometary plasma of comet 67P, and (ii) to characterize and map the Langmuir waves measured in the cometary plasma. A useful byproduct of this study is a comparison between the active and passive measurements of the RPC-MIP instrument, which gives some feedback on the density measurement capabilities of RPC-MIP.

The paper is organized as follows. Section 2 describes the RPC-MIP instrument and the electric field measurements taken by RPC-MIP while in passive mode. Section 3 introduces the method to extract the electric field emissions from the RPCMIP passive spectra. In Sect. 4 we cross-check the RPC-MIP passive measurements with the plasma density measurements taken by the RPC-MIP active mode to gain new insights into the instrument capabilities. In Sect. 5, we present evidence of Langmuir waves when the comet is interacting with solar wind disturbances like CMEs or solar wind stream interaction regions (SIRs). The possible mechanism exciting Langmuir waves during the passage of solar wind drivers are speculated in the discussion (Sect. 6) before the conclusions (Sect. 7).

\section{Instrumentation and theory associated to Langmuir waves in a cometary plasma}

The RPC-MIP active modes were used to study the plasma bulk properties during the Rosetta mission, and in particular the cometary plasma density (Henri et al. 2017; Hajra et al. 2017) and temperature (Gilet et al. 2017; Wattieaux et al. 2019). The main dataset when RPC-MIP was operating in active mode consists of mutual impedance power and phase spectrograms. The plasma frequency as well as the plasma density can be extracted from these mutual impedance spectra. The RPC-MIP active mode operated in the frequency range $7-3500 \mathrm{kHz}$ with various frequency resolutions. In active mode, the RPC-MIP was operating either in Short-Debye-Length (SDL) or Long-DebyeLength (LDL) modes that were designed to measure the plasma frequency in different plasma conditions. In addition, the SDL mode operated in four different submodes $\left(E_{1}, E_{2}\right.$, Anti-Phased, and Phased), which were used during different time periods of the mission. The most used submodes were Anti-phased and Phased (Gilet et al. 2020). A more detailed description of the RPC-MIP active measurements can be found in Trotignon et al. (2007) and Gilet et al. (2020).

While the bulk plasma characteristics, such as the plasma density and temperature, were studied by the active mode, the passive mode only used the receiving antennas to monitor the natural plasma waves in situ through their electric field counterpart, and in particular its component along the direction of the instrument, in the operating frequency range of RPC-MIP. As the RPC-MIP passive mode is only using the receiving antennas, it does not have similar submodes to those of the active mode (i.e., SDL or LDL) which can measure different density ranges that depend on the distance between transmitters and receiving antennas.

In the Earth's magnetosphere, the RPC-MIP frequency range encompasses the electron cyclotron frequency, which is proportional to the DC magnetic field amplitude, and electron Bernstein waves (Beghin et al. 2017). In the 67P cometary environment and in the interplanetary environments, the RPC-MIP frequency range was much higher than the electron cyclotron frequency within the electrostatic range. This is because of the much lower DC magnetic field amplitude in these environments. In the cometary ionized environment, the RPC-MIP passive mode is assumed to detect an electric field emission near the plasma frequency. The assumption is validated in Sect. 4 by comparison with the RPC-MIP active mode dataset.

Langmuir waves are plasma oscillations carried by electrons at a frequency close to the plasma frequency. When the ion dynamics has been neglected and the electron distribution is Maxwellian, the real part of the dispersion relation for the Langmuir waves in the long wavelength limit reads:

$\omega^{2}=\omega_{\mathrm{p}}^{2}\left(1+3 k^{2} \lambda_{\mathrm{D}}^{2}\right)$

where $k$ is wave vector, $\omega_{\mathrm{p}}$ is the angular plasma frequency for electrons, and $\lambda_{\mathrm{D}}$ is the Debye length. The imaginary part of the frequency describes the Landau damping (Landau 1946), still in a Maxwellian plasma, and reads:

$\gamma=\left(-\frac{\pi}{8}\right)^{1 / 2} \frac{\omega_{\mathrm{p}}}{\left|k^{3} \lambda_{\mathrm{D}}^{3}\right|} \exp \left(-\frac{1}{2 k^{2} \lambda_{\mathrm{D}}^{2}}-\frac{3}{2}\right)$.

Landau damping is the collisionless plasma process that attenuates electrostatic waves. If the term $3 k^{2} \lambda_{\mathrm{D}}^{2}$ increases in Eq. (1), for example due to an increase in the electron thermal speed or a decrease in the wavelength, the Landau damping increases rapidly. The Landau damping is negligible only for real frequencies close to the plasma frequency.

Taking this into account, we can expect the electric field emissions at the RPC-MIP passive spectra to occur at small wave vectors (i.e., at large wavelengths) near the plasma frequency. 
Otherwise, it is likely they would have been quickly damped in the cometary plasma because of the high Landau damping rate.

On the other hand, it is likely that the RPC-MIP passive mode is measuring electric field emissions whose source region is near to the region where they are observed. This assumption is based on the idea that the Langmuir waves emitted far away from the instrument would likely never reach the instrument because of the large density gradients (Edberg et al. 2015) near the comet.

Indeed, as the frequency of the Langmuir wave is conserved when it propagates through inhomogeneous plasmas, its wavelength varies. Therefore, first, if the Langmuir waves were generated in a plasma with a higher plasma frequency than that found near to the instrument, the wavelength would decrease through linear mode conversion while the wave is propagating, strongly increasing the Landau damping. Second, if the wave is generated in a plasma with a lower plasma frequency than that found near the instrument, it should propagate in a plasma where the plasma density is increasing. However, the Langmuir wave has a cut-off at the plasma frequency and the waves at frequencies smaller than the local plasma frequency are not able to propagate. Therefore, this second scenario would prevent the waves from arriving at the instrument as the waves would likely be reflected before reaching the spacecraft. Thus, in both cases, it is very unlikely that a wave generated far away would ever reach the instrument and we hereby conclude that the source of the electric field emissions observed by RPC-MIP passive mode is in the close vicinity of the instrument.

It is worth mentioning however that the electron plasma environment near comet $67 \mathrm{P}$ is not Maxwellian, as noted by several authors (Clark et al. 2015; Broiles et al. 2016a,b; Myllys et al. 2019). Instead, the plasma consists of several different electron populations. The electrons that originate from ionization processes, mostly photoionization, form the core of the electron population. A fraction of the core electrons are cooled down because of collisions with neutrals near the cometary nucleus (Eriksson et al. 2017; Wattieaux et al. 2019; Gilet et al. 2020) to add a colder electron population, especially prominent near perihelion. In addition, the electron distribution function shows a pronounced high-energy tail that consists of accelerated electrons that were measured near the comet throughout the mission (Clark et al. 2015; Myllys et al. 2019).

The existence of this high-energy tail in the distribution function must be taken into account to estimate the Landau damping near the comet. First, Thorne et al. (1991) studied the Landau damping of Langmuir waves in a hot, isotropic, unmagnetized and generalized Lorentzian plasma. The results were compared with Maxwellian plasma. The authors found that the damping of the Langmuir waves at long wavelengths becomes stronger in plasmas with a high-energy tail and the waves are even more clearly localized just above the plasma frequency compared to the Maxwellian case. Second, Rose et al. (2005) studied the Landau damping of electrostatic waves when the electron distribution consists of a dense core electron population and a low-density suprathermal electron distribution. Similar to Thorne et al. (1991), the authors observed that the attenuation of the Langmuir waves becomes stronger as the plasma becomes less Maxwellian and the importance of the suprathermal part with respect to the total density increases. Hence, we further expect the RPC-MIP passive mode to detect electric field emissions just above the plasma frequency.

\section{Extracting the electric field signatures and the plasma frequency using the RPC-MIP passive mode}

The RPC-MIP passive dataset consists of electric field spectrograms where the measured 1D electric field amplitude is expressed as a function of time and frequency. The frequency ranges from 7 to $3584 \mathrm{kHz}$ (resp. $7-448 \mathrm{kHz}$ ) in the so-called FULL (resp. WINDOW) submode. When electric field oscillations related to Langmuir waves are observed, the spectral amplitude displays a peak near the electron plasma frequency. Hence, the plasma frequency can be determined from the RPC-MIP passive spectrograms by localizing the maximum power in each time-step when Langmuir waves are present in the plasma.

However, there are several interferences in the RPC-MIP spectrograms that can have higher power amplitude than the real electric field emission. The locations of the interferences (i.e., the frequency band) stay almost fixed in time. Thus, the interferences can be recognized from the spectra as horizontal lines with high-power amplitude. The interferences are caused by the on board electronics and their amplitude has been observed to vary with the temperature of the RPC-MIP preamplifier. These instrumental artifacts have been taken into account in this work.

\subsection{Search method for the plasma frequency}

To extract the plasma density from the RPC-MIP passive dataset, we created an automated algorithm that detects the electric field emissions from the RPC-MIP electric field (passive) spectrograms to get the plasma frequency. The starting point for the electric field emission detection is the electric field spectrograms that contain the power amplitude as a function of time and frequency. The spectrograms are treated in one-day intervals. Because of the interferences in the spectra, it is not possible to observe the natural electric field emissions in all frequency channels. For instance, the frequency band at $147 \mathrm{kHz}$ was excluded from the study because the band was strongly contaminated by interference.

The electric field emission-detection routine first identifies the maximum amplitude for each individual time-step. The amplitude and the frequency at which the maximum was located are saved. In an attempt to make sure that the amplitude maximum is not background noise or an interference, we require that it fulfill certain criteria. First, the location of the maximum amplitude should be unambiguous: spectra characterized by two or more disjoint frequencies with the same maximum amplitude value are discarded from the analysis. Second, the peak amplitude must be sufficiently above the background noise level. The background level was estimated for each frequency band separately over an entire day by computing the median power. The power amplitude for the electric field emission has to be at least three times higher than the median. For the frequency bands that are the most contaminated by interference, we require that the power amplitude be ten times higher for it to be selected as an emission.

Two example spectrograms for the RPC-MIP passive mode are shown in Fig. 1. The power is given as a function of time and frequency. The upper panel in Fig. 1 is an example of a time interval when no electric field emissions were detected. The horizontal stripes in the spectrogram are interferences. The lower panel shows an interval when clear electric field emissions were detected. 

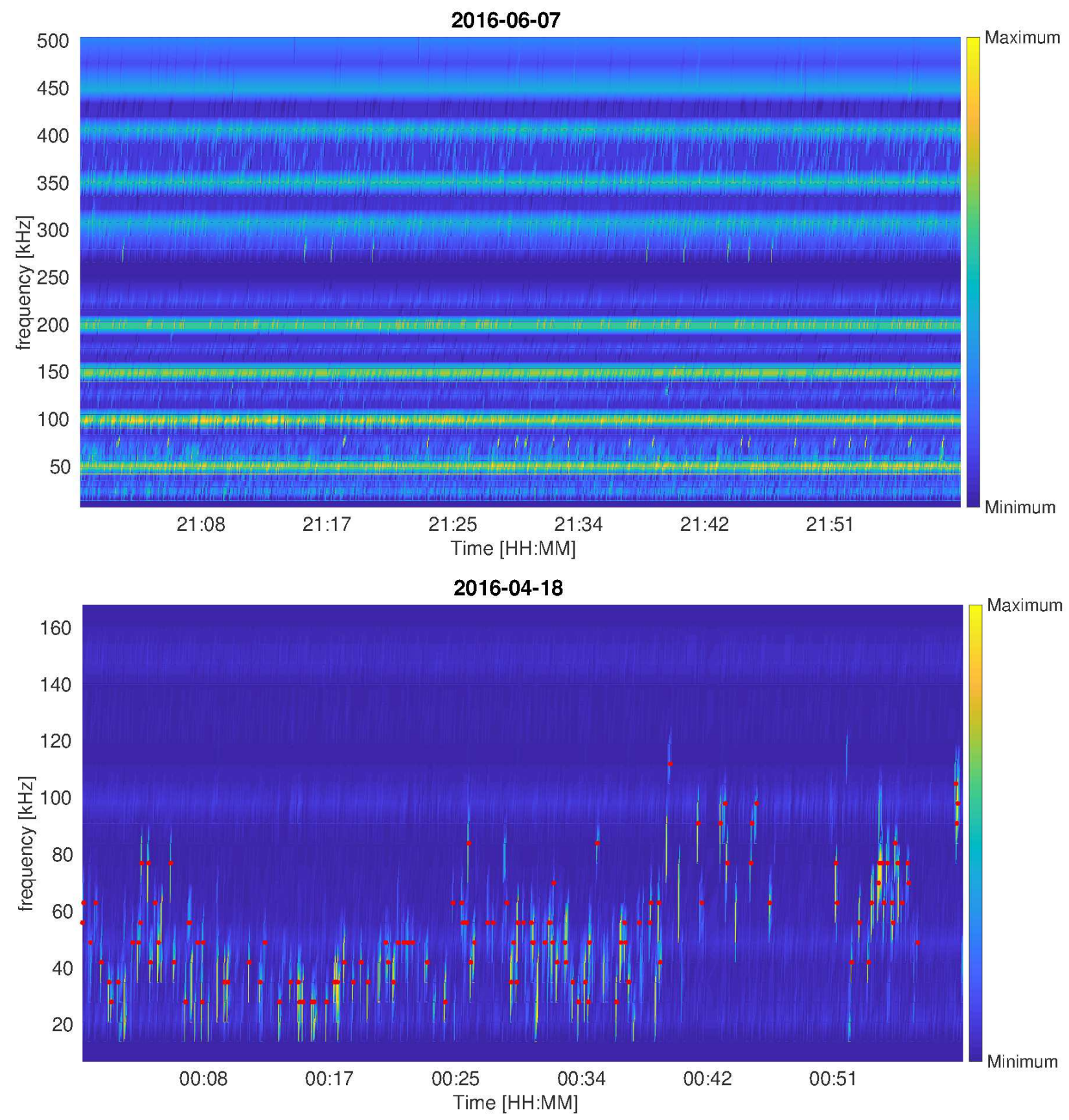

Fig. 1. Examples of the RPC-MIP passive spectrograms. Upper panel: typical passive spectrogram that is characterized by multiple interferences. Lower panel: time interval featuring many strong electric field emissions. The red dots show the locations of the emissions identified by the search algorithm. The spectrograms have been normalized so that the color scales from minimum to maximum in order to ease the identification of interferences or electric field emissions in the spectrograms.

The method explained above is designed to detect most of the highest amplitude electric field emissions and to avoid misinterpreting various instrumental artifacts such as interferences. However, it is likely that a fraction of the emissions are not detected and, conversely, that a small fraction of the detection contains signals that correspond to interferences.

\subsection{Electric field measurements at the plasma frequency with RPC-MIP passive mode}

The RPC-MIP passive mode observed electric field emissions throughout the time interval when Rosetta was escorting comet
67P. Our search routine detected an emission in around $1 \%$ of all the measured passive spectra, indicating that electric field emissions at the plasma frequency were not continuous in the cometary plasma, but appeared sporadically.

The daily ratio between the number of spectra with identified electric field emissions $\left(N_{\text {emission }}\right)$ and the total number of passive spectra $\left(N_{\text {spectra }}\right)$ is shown for the whole cometary operation of Rosetta as a histogram in Fig. 2. The passive emissions during 2014 are relatively rare compared to other years but this is partly affected by the lower quality of RPC-MIP passive spectra during that year. Indeed, during 2014 the spectra were noisier and contained stronger interferences that made it difficult to 


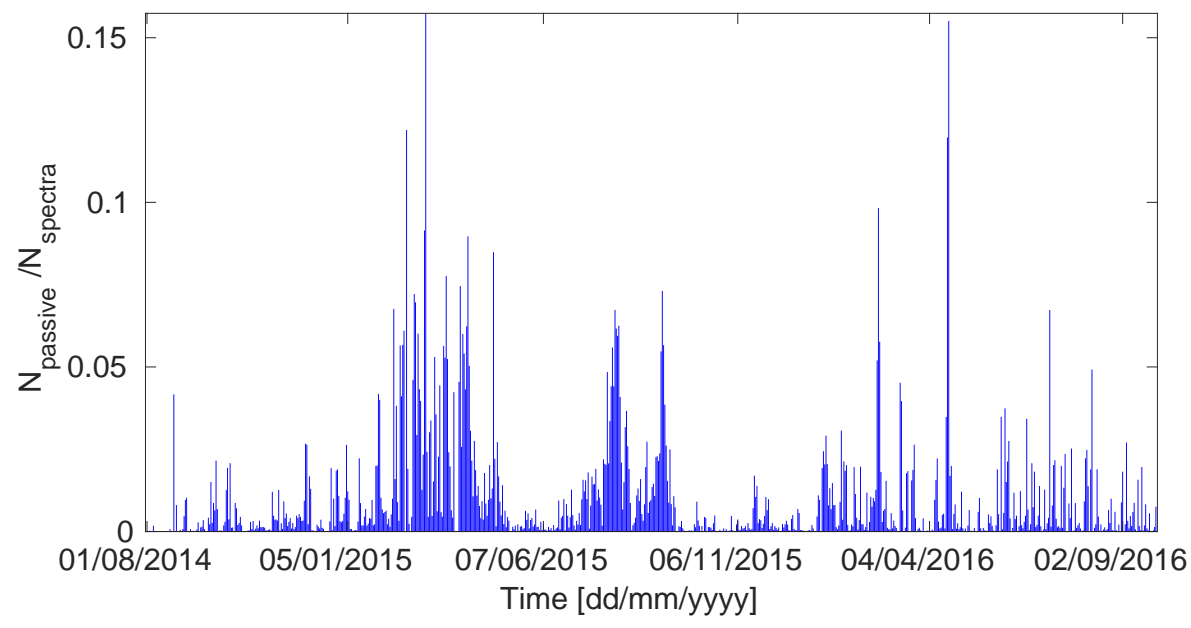

Fig. 2. Daily occurrence rate of electric field emissions in the RPC-MIP passive dataset, defined as the ratio of the number of spectra showing signatures of electric field emissions $\left(N_{\text {emissions }}\right)$ to the number of all measured passive spectra $\left(N_{\text {spectra }}\right)$. extract the electric field emissions. The majority of the passive emissions occur near perihelion (July to September 2015) and during March and April 2015.

Temporal variability of the occurrence rate of the electric field emissions is expected to be related to the variability of the sources causing Langmuir waves. The Langmuir waves can be caused by several factors that are either related to some nearcomet processes (e.g., the presence of cold cometary electrons, as discussed in Sect. 6.3) or to some external drivers, like largescale solar wind structures (e.g., SIRs, studied in Sect. 5.1, or CMEs, in Sect. 5.2).

\section{Comparison between the plasma density defined from RPC-MIP mutual impedance (active) and electric field (passive) measurements}

In this section, we make use of the electric field detection method described above, assuming that the electric field emissions occur at the local plasma frequency, to extract an estimate of the plasma density. The plasma densities extracted from the RPC-MIP electric field (i.e., passive) spectra are cross-checked with the plasma densities extracted from the RPC-MIP mutual impedance (i.e., active) spectra, which are available at the ESA Planetary Science Archive (PSA). In the following, we refer to "active" (resp. "passive") density, which is the plasma density obtained from the RPC-MIP mutual impedance (resp. electric field) spectra.

\subsection{Comparison between the active and passive measurements}

As active and passive RPC-MIP spectra are not sampled simultaneously, the comparison between active and passive density measurements has been made by considering nearby measurements, discarding cases when no plasma measurement from the RPC-MIP mutual impedance (active) spectra is made within $32 \mathrm{~s}$ of the detected electric field (passive) emission. It is not always possible to compare the passive densities with the active ones because not all mutual impedance (active) measurements allow the plasma density to be extracted.

If the difference between the passive and active densities is smaller than the measurement uncertainties, the two density estimates are considered as matching. The density uncertainties for the passive detections are defined as the width of the frequency band. Because of the coarse frequency resolution of the passive mode, the consecutive plasma density measurements extracted from the RPC-MIP electric field spectra can be aligned if the density variations in the cometary plasma are inside the width of the frequency bin (i.e., inside the error bar). The uncertainties for the active measurements are available on the ESA PSA and information on the active measurements and their uncertainties can be found from the RPC-MIP User guide prepared by the Henri \& RPC-MIP team (2018). If the passive density does not agree with the nearest active density, it is further compared with the second nearest active density that is still within the 32-s time window. In the highly dynamical cometary plasma, we regularly observed cases in which the active plasma density rapidly changes while the passive density lies between these two different but consecutive active density measurements. In these cases, the passive density is also interpreted as agreeing with the active measurements because it is consistent with the spatial and temporal evolution of the plasma density.

Most of the time, the active and passive densities match each other. When RPC-MIP is operating in LDL mode, the active and passive densities are found to match in only $60 \%$ of cases, while the matching rate is $67 \%$ for the SDL mode. The reason that the densities are more closely matching when the SDL mode is operated is well known: The LDL mode can indeed only measure active plasma densities up to $300 \mathrm{~cm}^{-3}$, simply because of the limited frequency range operated in this mode. However, the electric field spectra in LDL mode do not have such a strong limitation, and so passive density measurements can capture the plasma density even when it exceeds the LDL active density threshold. We conclude that (i) the LDL operational mode is not suitable for comparing active and passive density measurements and (ii) passive density measurements are of great added value in the LDL mode to reach plasma densities above the LDL active density limit.

On the other hand, when RPC-MIP is operating in the SDL mode, the limitation associated to the LDL mode is not present. The RPC-MIP SDL mode was used in four submodes, but for the most part it operated either in anti-phased or phased submodes (Gilet et al. 2020) and therefore we only focus on these two. If we only consider the SDL submode called phase (resp. anti-phase), $75 \%$ (resp. $61 \%$ ) of the densities match, while $25 \%$ (resp. $40 \%$ ) do not match. The reason leading to the lower matching rate in the SDL anti-phase compared to the SDL phase submode is discussed in the following section.

\subsection{Differences between the active and passive measurements in SDL}

The discrepancies between the active and passive plasma densities in SDL modes are shown in Fig. 3. The solid lines (resp. 


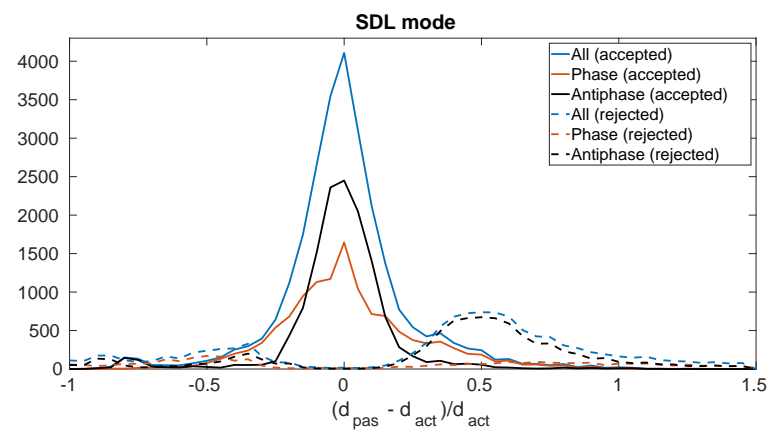

Fig. 3. Relative error between the RPC-MIP active $\left(d_{\text {act }}\right)$ and passive $\left(d_{\text {pas }}\right)$ densities. The distributions indicated with solid lines show the relative difference when the difference between the RPC-MIP active and passive densities were smaller than the measurement uncertainties (accepted), while the dashed lines indicate times when the density difference was larger than the uncertainties (rejected).

dashed lines) show distributions of the relative mismatch for time periods when both densities are considered to be matching (resp. not matching). The blue color shows the analysis for all SDL submodes combined, while the analysis performed considering only the SDL anti-phase (resp. phase) submode is shown in red (resp. black).

Figure 3 shows that the distributions have a clear dependence on the SDL operational submode. On the one hand, in phase submode the distribution for the mismatching densities (red dashed line) is bimodal, with two peaks on both sides of zero that have almost the same amplitude. This indicates that no particular systematic discrepancy is observed between both density estimates. On the other hand, in anti-phase submode the distribution of the mismatching densities (black dashed line) instead is dominantly localized on the positive side. This means that the plasma density estimated from the RPC-MIP electric field (passive) measurements is higher than the plasma density estimated from the RPC-MIP mutual impedance (active) measurements in anti-phase around $33 \%$ of the time. This might reflect the fact that the SDL anti-phase active density measurements can occasionally underestimate the plasma density by up to about $50 \%$ compared to the passive plasma density estimates.

We now focus on the origin of the higher mismatch observed in anti-phase submode. By only considering the SDL anti-phase and the times when the active and passive plasma densities do not match, and studying how the relative mismatches are distributed for different density ranges (not shown), we find that the relative error becomes predominantly positive when the active plasma density exceeds $700 \mathrm{~cm}^{-3}$.

Two examples of density time series are shown in Fig. 4, with two different time intervals when the RPC-MIP was operating in SDL anti-phase submode. The first (resp. second) example illustrates the density match (resp. mismatch) distribution shown with the black dashed line (resp. black solid line) in Fig. 3, when RPC-MIP was operated in anti-phase submode. During the first interval the active and passive density time series match well (upper panel), while during the second interval the passive density time series is seen to be systematically located above the active densities (lower panel). However, in this second case, both densities show similar temporal evolution. This second case indicates that the high amount of positive mismatch shown in SDL anti-phase in Fig. 3 can hardly be associated with false density detection related to interferences. The possible reasons for this higher mismatch rate are discussed in the following sections.

\subsubsection{Short-Debye-Length anti-phase and cold electrons}

The time intervals shown in Fig. 4 are taken near perihelion. The heliocentric distance of comet 67P during these time intervals was 1.25 $\mathrm{AU}$ (upper panel) and 1.29 AU (lower panel), and so the neutral outgassing conditions during both time intervals were similar. On the other hand, the first time interval, taken from August 2015, shows density measurements above the northern hemisphere of the comet while the second time interval, taken in September of the same year, shows density measurements from the southern hemisphere. The plasma density during the example events varies between 1000 and $5000 \mathrm{~cm}^{-3}$.

The RPC-LAP instrument (Eriksson et al. 2007) observed signatures of cold electrons during both time intervals. The RPCMIP can also be used to detect the presence of cold electrons in the cometary environment because the two-temperature electron plasma can cause additional resonance peaks to the mutual impedance probe power spectrum (Gilet et al. 2017). Indeed, in a two-temperature plasma, two electrostatic wave modes can exist: Langmuir waves with thermal correction and electron acoustic waves (Gilet et al. 2017). The resonance peak at lower frequencies is related to the electron acoustic branch and the peak is located near the cold plasma frequency, while the second peak corresponds to the total plasma frequency caused by Langmuir waves. The RPC-MIP instrument uses this as a principle to observe the existence of cold electrons (Gilet et al. 2020). A detailed description of the cold electron detection method using the RPC-MIP instrument is given by Gilet et al. (2020).

RPC-MIP also observed cold electrons (i.e. double resonance peaks in the mutual impedance spectra) throughout the August 2 interval shown in Fig. 4, while RPC-MIP did not detect any cold electrons on the September 8, 2015, using this method. As RPC-LAP observed cold electrons even during the September 8 interval, it seems that the RPC-MIP instrument was not able to detect the cold electron population because of instrumental detection properties. Indeed, it is known that RPC-MIP is not always able to observe the presence of cold electrons and this is especially true during the SDL anti-phase mode (Gilet et al. 2020).

Gilet et al. (2020) executed a statistical study of cold electron observations by RPC-MIP around comet 67P. The majority of cold electrons, that is, $94.79 \%$ of all the cold electron observations, were observed when RPC-MIP was operating in SDL phase submode. This is in agreement with the simulations by Wattieaux et al. (2019), who modeled the mutual impedance spectra for RPC-MIP using the phase and anti-phase submodes. The two resonances were more easily detectable from the mutual impedance spectra when the spectra were generated using the phase submode.

In the presence of cold electrons, the second resonance peak (i.e., the peak for plasma frequency) in the SDL anti-phase spectrum can be flattened and is not detectable (see the Appendix in Gilet et al. 2020). Thus, it is possible that RPC-MIP is capturing the cold electron frequency instead of the total one. As shown by Gilet et al. (2020), the shape of the RPC-MIP mutual impedance spectrum depends on the ratio between the cold and total electron density as well as the temperature ratio of the warm and cold electrons. For example, the SDL anti-phase submode may show a resonance peak at the cold electron frequency while the total plasma frequency peak is flattened. This happens when the warm electron density is dominating, the cold-to-total electron ratio is 0.6 or smaller, and the temperature ratio is between 60 and 150 . 

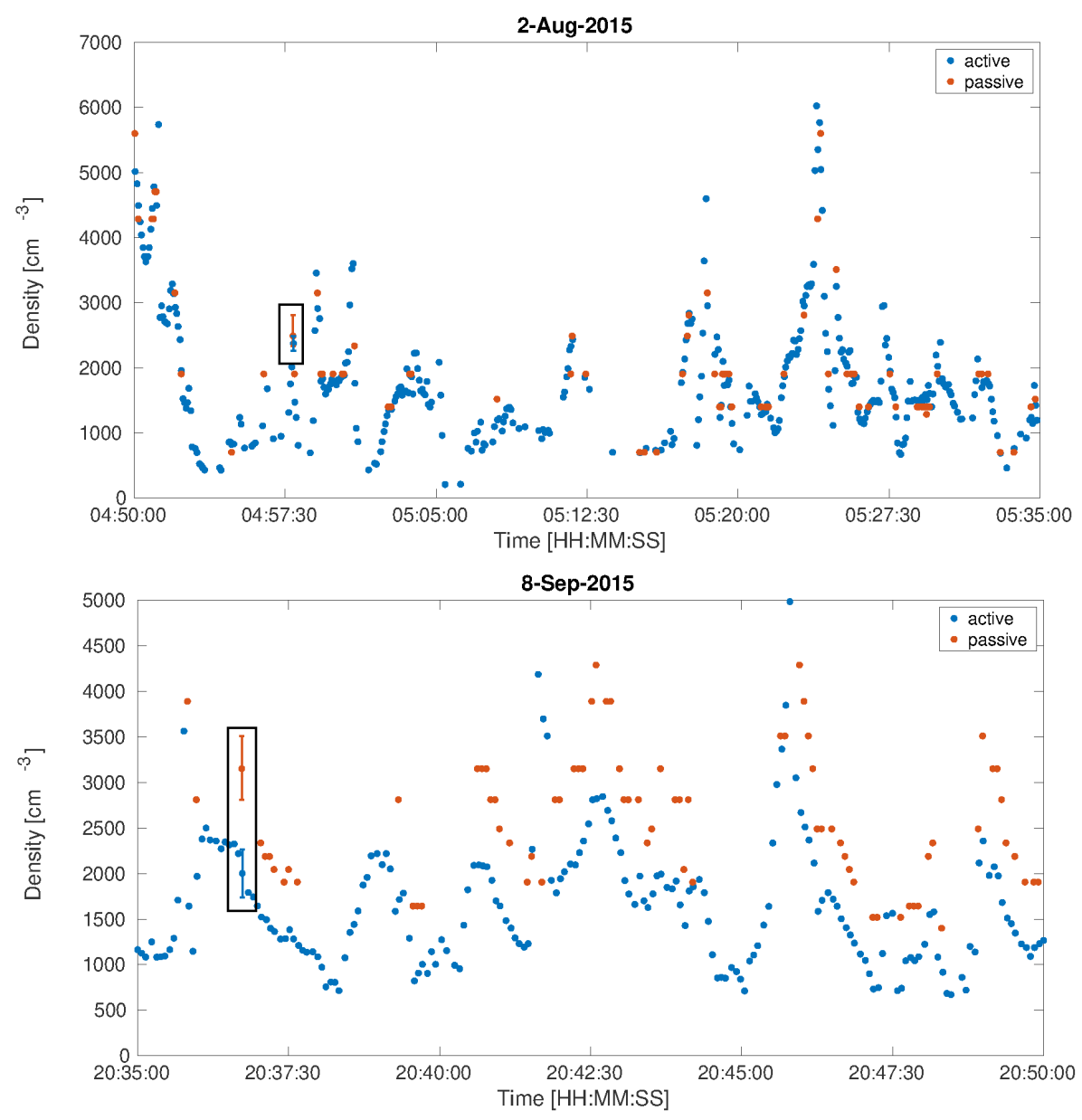

Fig. 4. Plasma density measurements derived from RPC-MIP active (blue) and passive (red) modes. The instrument was operating in SDL anti-phase submode. Both panels show examples of the measurement uncertainties for one passive and one active density observation; these are highlighted with black rectangles. The cometocentric distance of Rosetta was around $207 \mathrm{~km}$ on August 2, 2015, and $334 \mathrm{~km}$ on September 8, 2015.
If the cold electron density is dominating $\left(n_{\mathrm{c}} / n_{\mathrm{tot}}>0.7\right)$, the SDL anti-phase submode has only one resonance peak that corresponds to the cold plasma frequency, while the peak for the total plasma frequency is missing completely (see the Appendix of Gilet et al. 2020). If the cold-to-total electron density is above 0.9 , the cold electron frequency is more or less the same as the total plasma frequency. However, if the cold electron density decreases with respect to the total electron density, the cold electron plasma frequency starts to drop below the total plasma frequency, and as the instrument is not able to capture the resonance peak for the total plasma frequency, the RPC-MIP active mode underestimates the plasma density.

We can test the above-mentioned scenario. If we assume that the RPC-MIP passive mode is giving the correct plasma frequency (i.e., density) and the frequency given by the active mode is the cold plasma frequency, we can estimate the cold-to-warm density ratio from the lower panel of Fig. 4. The estimated ratio is shown in Fig. 5. During the studied time interval, the ratio is always above 0.6 , meaning that the cold electron density dominates. Based on the simulations by Gilet et al. (2020), this means that we are in the region where the only visible resonance peak in the SLD anti-phase submode corresponds to the electron acoustic frequency. Thus, this scenario can explain the difference in the densities.

To illustrate this discussion, Fig. 6 shows three examples of mutual impedance spectra (i.e., active measurements) during September 8, 2015, when the plasma densities given by the active and passive RPC-MIP measurements are different. For each panel, the location of the resonance peak is marked with a red line, while the location of the plasma frequency given

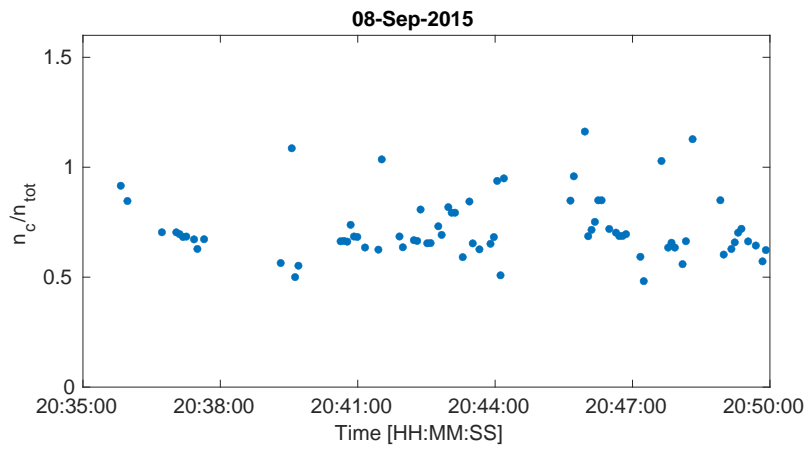

Fig. 5. Cold-to-total electron density ratio during September 8, 2015, at 20:35:00-20:50:00.

by the passive mode is indicated with a black line. The panels show three consecutive time-steps. The estimation of the plasma frequency from the active and passive models differs in each spectrum. Unlike the other spectra, the spectrum in the right panel shows a double resonance (i.e., two peaks). The second peak is near the plasma frequency given by the passive mode. Thus, it is likely that the first peak is related to the electron acoustic frequency while the second one is the peak for total plasma frequency. The sudden appearance of the total plasma frequency peak may reflect variations in the cold-to-total density or cold-to-warm electron temperature ratios.

So far, we have focused on the cause of the differences between the active and passive densities during the September 8, 2015, as shown in Fig. 4. However, during the August 2 the 


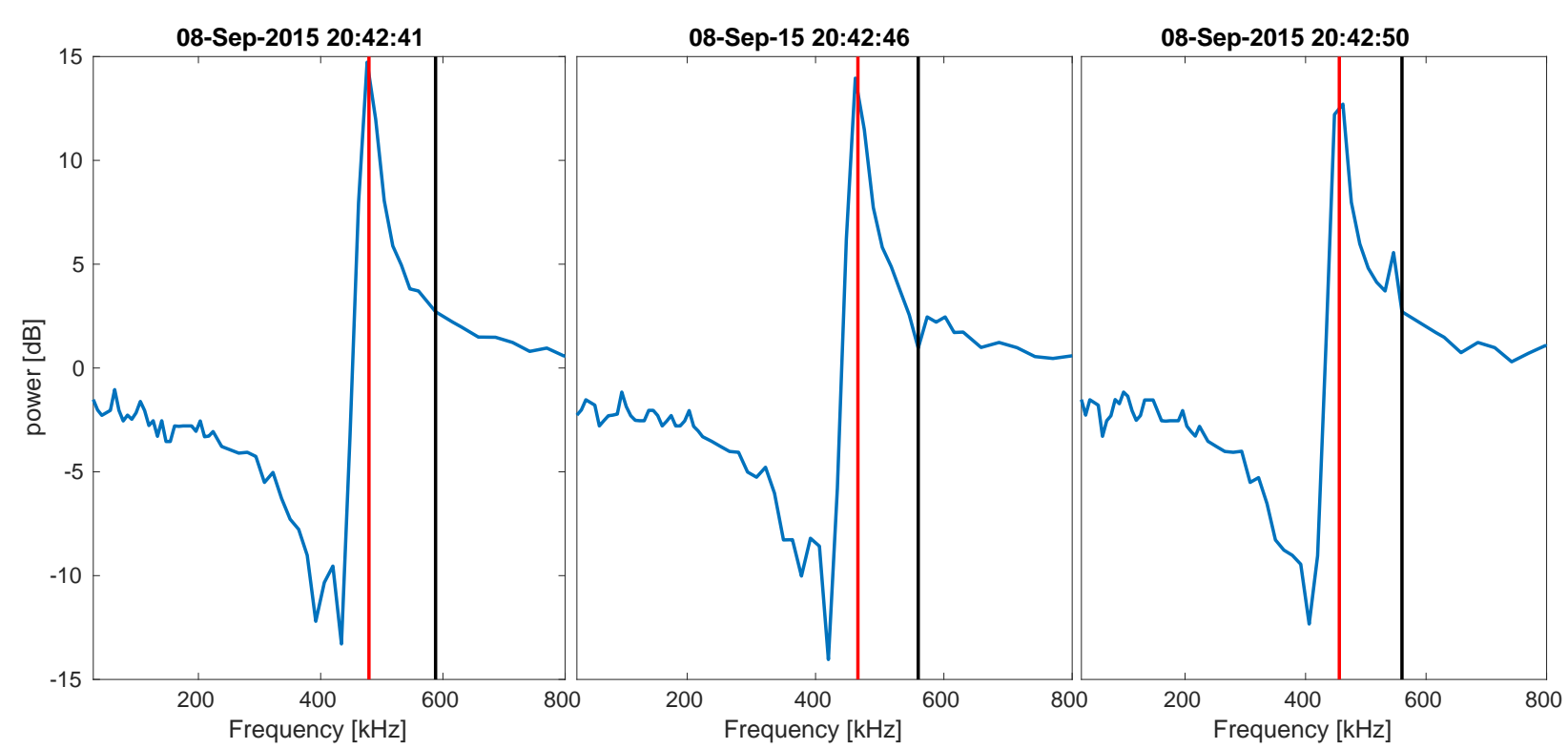

Fig. 6. RPC-MIP active spectra for three consecutive time-steps. The red line indicates the location of resonance maximum in the active spectra, while the black line is the maximum at the passive spectra.

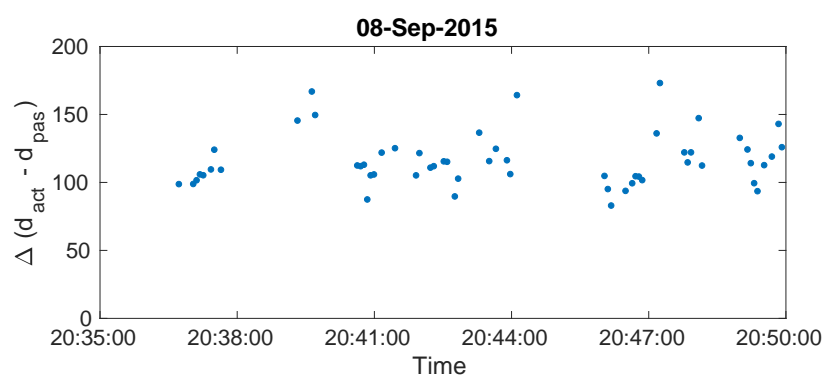

Fig. 7. Estimated distance between the source region of electric field emission measured by the RPC-MIP passive mode and the location of the spacecraft (i.e., the location of the active measurements) as a function of time.

passive and active densities agree well even though the RPCMIP was also operating in SDL anti-phase submode. We can only speculate on how the active mode was able to estimate the plasma frequency correctly during this time interval. As mentioned previously, the RPC-MIP observed several double peaks (i.e., cold electrons) during the time interval. Hence, it seems that the resonance peak corresponding to the total plasma frequency is visible with the peak corresponding to the electron acoustic branch, and therefore the algorithm extracting the total plasma frequency from the active spectra was able to select the correct peak.

As a concluding remark, we can add that when RPC-MIP is operating in SDL anti-phase submode, the plasma density may be underestimated during plasma conditions containing a lot of cold electrons. According to our study, this occurs more frequently close to perihelion and is scarcely observed far from perihelion. On the other hand, in these conditions the RPC-MIP instrument can sometimes offer estimates for both cold and total plasma densities.

\subsubsection{Location of the electron field emissions}

As explained in the previous section, the mismatch between the active and passive densities is most probably related to instrumental behavior, coupled to the actual composition of the plasma in terms of cold electrons. Nonetheless, there are other possibilities. For example, the difference could be related to the location of the source region of the electric field emission causing the passive detection.

The plasma density near the comet is inversely proportional to the radial distance $\left(r_{\mathrm{c}}\right)$ on the comet surface (Edberg et al. 2015; Galand et al. 2016). If the source region for the passive emission was nearer the comet than the spacecraft and the location where the active measurement was taken, the passive detection would give higher densities compared to the active one.

If we assume that the active measurements are giving the correct total plasma density near the instrument and the passive emissions originate from a region closer to the comet, we can estimate the distance that the Langmuir wave needs to travel before it is detected by RPC-MIP. As we know the distance of the spacecraft $\left(r_{\mathrm{SC}}\right)$ from the comet surface as well as the active and passive densities, we can estimate the source location $\left(r_{\text {passive }}\right)$ for the passive density using the known $1 / r_{\mathrm{c}}$ dependence. Thus, the following equation gives us the distance from the comet's surface where the Langmuir wave was emitted:

$r_{\text {passive }}=\frac{n_{\text {active }} r_{\mathrm{SC}}}{n_{\text {passive }}}$.

When we use the passive data points shown in Fig. 4 and compute the estimated location for the wave emissions, the distance between the active and passive measurements is around 100 $150 \mathrm{~km}$ (see Fig. 7). The distance is very large and it would be extremely unlikely that the waves could have reached the instrument without being damped. Therefore, we conclude that this scenario is unlikely, that the electric field emissions are very local phenomena, and that the source is likely to be very near to the instrument.

\section{Langmuir wave observations during solar wind transient events}

In the following sections, we focus on electric field measurements at the plasma frequency observed by RPC-MIP when transient solar wind structures, such as SIRs or CMEs, reached comet 67P. 
Table 1. Examples of days characterized by a high amount of electric field emission.

\begin{tabular}{cc}
\hline \hline Date & Driver \\
\hline $2015-02-10$ & SIR \\
$2015-02-15$ & $?$ \\
$2015-02-17-2015-02-18$ & SIR \\
$2015-02-20$ & SIR \\
$2015-02-26-2015-03-01$ & SIR and HSS \\
$2015-03-06-2015-03-07$ & CME and SEP event \\
$2015-03-14$ & SIR \\
$2015-03-23-2015-03-24$ & SIR? \\
$2015-04-03$ & $?$ \\
$2015-04-05-2015-04-06$ & $?$ \\
$2015-04-08-2015-04-10$ & $?$ \\
$2015-04-29$ & $?$ \\
$2015-07-31$ & $?$ \\
$2015-08-02-2015-08-05$ & $?$ \\
$2015-09-07-2015-09-09$ & SIR and HSS \\
$2016-02-23-2016-02-25$ & SIR (Sect. 6) \\
$2016-03-12-2016-03-13$ & SIR (Sect. 6) \\
$2016-03-17-2016-03-18$ & SIR (Sect. 6) \\
$2016-03-21-2016-03-24$ & SIR \\
$2016-04-18-2016-04-19$ & SIR (Hajra et al. 2018) \\
$2016-06-14$ & SIR (Hajra et al. 2018) \\
$2016-07-07$ and 11-07-2016 & SIR (Hajra et al. 2018) \\
$2016-08-04$ &
\end{tabular}

\subsection{Langmuir wave observations during SIR interactions}

A number of intense electric field emissions at the plasma frequency observed by RPC-MIP in passive mode (as shown in Fig. 2) appeared to occur during days when the comet was interacting with SIRs. Solar wind stream interaction regions are commonly referred to as corotating interaction regions (CIRs) in the literature if they are repeated after 27 days (i.e., after one solar rotation). Studying the repetition of SIRs is beyond the scope of this study, and therefore we globally use the term SIRs (which includes CIRs) in this paper. Table 1 lists (i) the days that are characterized by a strong electric field activity at the plasma frequency (Fig. 2) and (ii) their possible associated transient solar wind drivers arriving at the comet 67P. We have also added the SIR events studied by Hajra et al. (2018) to the Table 1 if they were driving electric field emissions.

We note that during April 2015 the solar wind ions started to disappear from the RPC Ion and Electron Sensor (RPC-IES; Burch et al. 2007) and Ion Composition Analyser (RPC-ICA; Nilsson et al. 2007b) data. During the time interval when Rosetta was not able to measure the solar wind, the spacecraft was located inside the so-called solar wind cavity (Nilsson et al. 2017a). Solar wind data did not re-appear until December 2015 (Nilsson et al. 2016), and therefore it was not possible to unambiguously identify the possible solar wind drivers of events occurring during this time interval. We highlight that Langmuir waves can also be caused by some near-cometary processes - for example related to cold electrons, as speculated in the discussion section - that are not related to solar wind.

We first focus on SIR impacts at comet 67P. Edberg et al. (2016a) studied SIR events near 67P during pre-perihelion in 2014, while Hajra et al. (2018) investigated SIRs during postperihelion in 2016. Both studies reported that, during SIR interactions with comets, the flux of suprathermal electrons
$(10-100 \mathrm{eV})$ in the cometary environment is enhanced and increased in energy. We identified the possible SIR drivers from the data by looking for signatures similar to those reported by Edberg et al. (2016a) and Hajra et al. (2018), such as solar wind proton velocity discontinuities, enhanced suprathermal electrons, magnetic field fluctuations, and increases in flux and energy of cometary water ions. In addition, we searched for the most likely counterparts for the SIRs at $1 \mathrm{AU}$ and estimated their arrival time to comet $67 \mathrm{P}$ by co-rotating the structures using the methods reported in Appendix A.

We have chosen to focus on three of the four SIR intervals studied by Hajra et al. (2018), who examined four SIR impacts on comet 67P during the last four months of the Rosetta mission, as examples to show their effect on electric field emissions at comet 67P. The September 2016 SIR event studied in Hajra et al. (2018) was not included in Fig. 8 as it only shows a very weak electric field wave activity at the plasma frequency. The pre-perihelion SIR events studied by Edberg et al. (2016a) occurred during 2014, when the quality of the RPC-MIP electric field spectra was low because of the low signal-to-noise ratio that impeded our study of those events.

The passive density together with the density obtained from the RPC-MIP mutual impedance measurements (active) are shown in Fig. 8 for the June, July, and August 2016 SIR events studied by Hajra et al. (2018). The vertical black lines correspond to the forward and reverse shocks that are bounding the SIRs. All the SIR intervals in Fig. 8 show Langmuir wave emissions during the stream interface. Moreover, the July 2016 SIR also shows emissions after the reverse wave during the high-speed stream interval following the structure. In addition to the density plot, an example of the electric field spectrogram measured by the RPCMIP passive mode during the July 2016 SIR event is shown in Fig. 9.

Three additional examples of SIR impacts on comet 67P, which are characterized by intense and frequent electric field activity at the plasma frequency, are shown in Fig. 10. The first SIR occurred approximately between March 12, 2016, and March 14, 2016, the second between March 17, 2016, and March 19, 2016, and the third between March 21, 2016, and March 23, 2016. The possible counterparts for the SIR periods at $1 \mathrm{AU}$ are shown in Appendix A. At $1 \mathrm{AU}$, the speed difference between the slow and fast solar wind associated with these three SIR intervals is only around $300 \mathrm{~km} \mathrm{~s}^{-1}$ and the maximum speed for the highspeed stream was around $650 \mathrm{~km} \mathrm{~s}^{-1}$. The SIRs are not separated by $\sim 27$ days, the solar rotation period. Hence, the streams are presumably originating from different source regions on the Sun.

The top three panels a-c in Fig. 10 show the density, temperature, and speed for the solar wind protons in the near-comet environment. The solar wind moments have been extracted from the RPC-ICA measurements. Panel d shows the X-component for the solar wind ion velocity in the cometocentric solar equatorial (CSEQ) reference frame. In this frame, the $x$-axis is parallel to the Sun-comet line, pointing towards the Sun. The $z$-axis is pointing towards the solar north pole and the $y$-axis completes the right-handed coordinate system. Thus, panel d shows whether the solar wind protons are predominantly flowing antisunward $\left(v_{X}<0\right)$ or sunward $\left(v_{X}>0\right)$, as a result of their interaction with the cometary induced magnetosphere. When cometary ions are produced by ionization processes in the coma, they get picked up by the interplanetary electric field and the solar wind gains more mass. This process is known as massloading. The mass-loading is deflecting the flow direction (Behar et al. 2016). If the deflection is strong enough, ultimately the solar wind starts flowing back towards the Sun. 


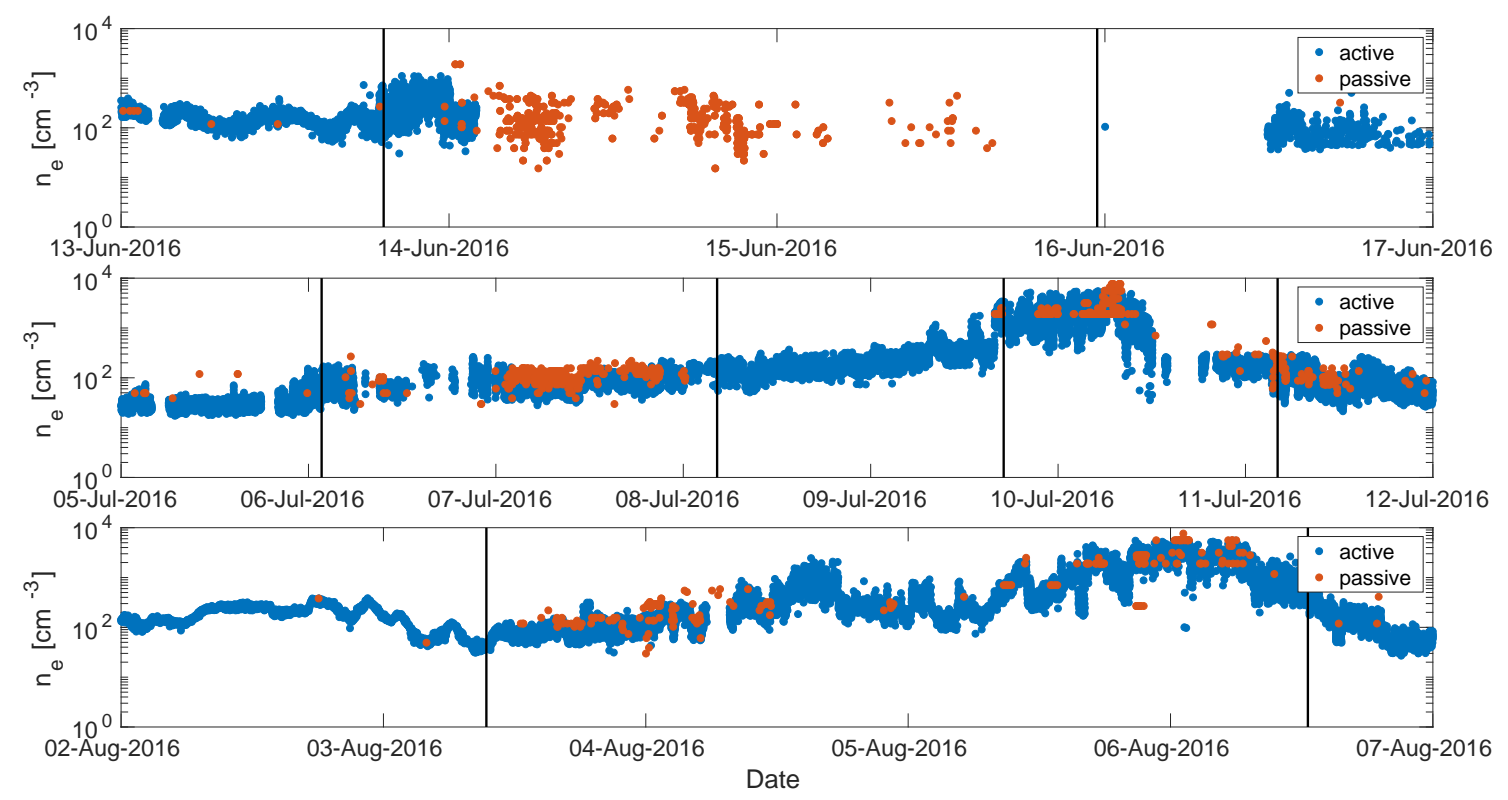

Fig. 8. Three SIR intervals studied by Hajra et al. (2018). The blue dots show plasma density extracted from the RPC-MIP active measurements, while the red dots are from the RPC-MIP passive mode.

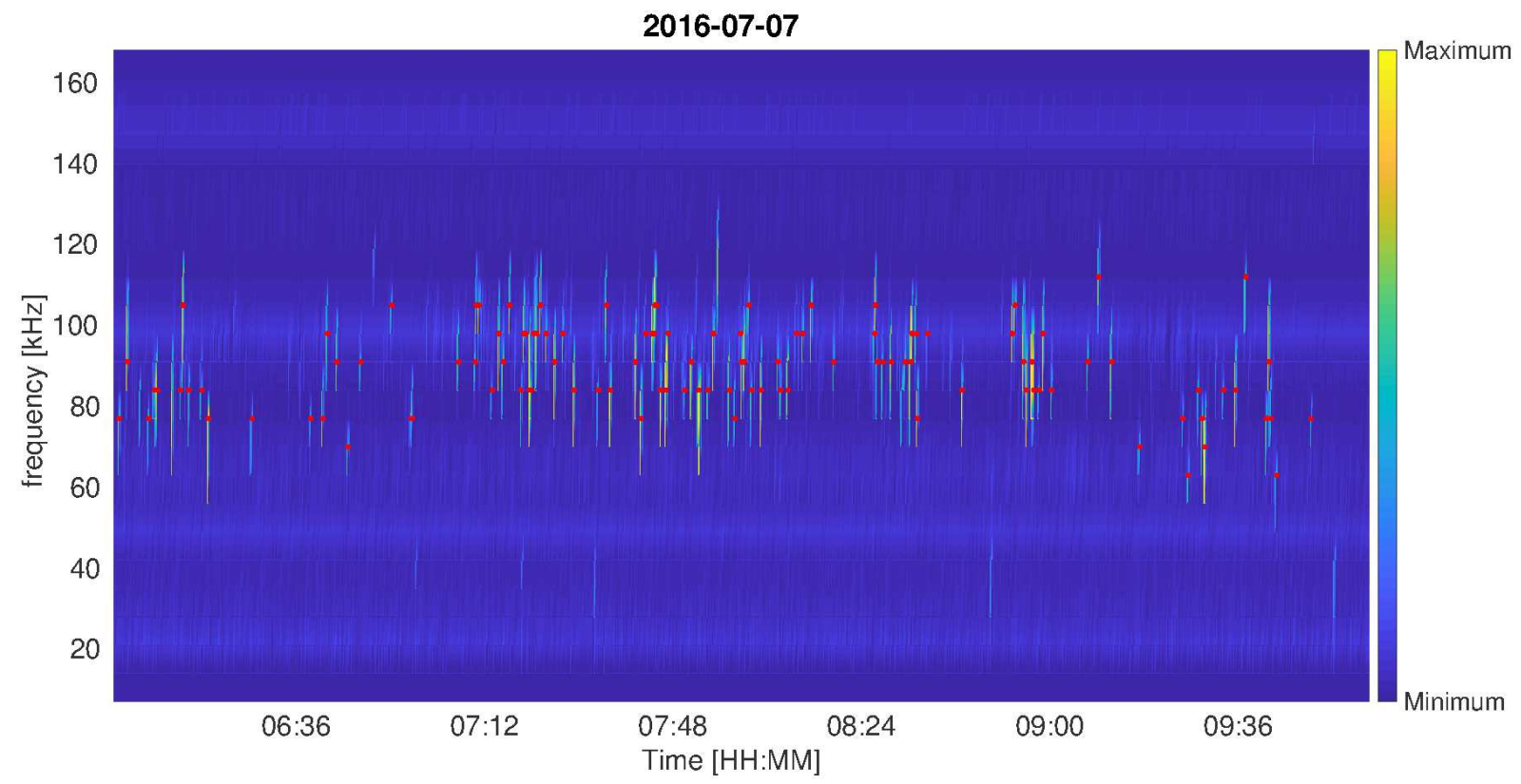

Fig. 9. Example spectrogram showing electric field emissions measured by the RPC-MIP passive mode during the July 2016 SIR event studied by Hajra et al. (2018). The red dots indicate the frequency of electric field emissions identified at the plasma frequency. The spectrum has been normalized so that the color scale goes from minimum to maximum power amplitude.

Panel e in Fig. 10 shows ion measurements from RPC-IES. The ion counts have been summed over all measured directions. The light green signal near energies of $10^{3} \mathrm{eV}$ indicates solar wind protons, while the paler signal at slightly higher energies is caused by heavier helium ions. The enhanced counts at low energies are related to cometary pick-up ions. Panel $\mathrm{f}$ is showing the electron counts from RPC-IES summed over all directions. Finally, the bottom panel g shows the total (i.e., cometary and solar wind) electron density extracted from the RPC-MIP mutual impedance measurements (i.e., active mode, blue dots) and the electron density extracted from the RPC-MIP electric field measurements (i.e., passive mode, red dots) during the time-steps with significant electric field emissions at the plasma frequency. The SIR intervals can be recognized from the solar wind features like enhanced solar wind proton density and temperatures. The solar wind proton velocity also shows a mild increase (panel c). The magnitude of the solar wind proton velocity shows scatter during the interaction with the SIRs. The scatter is believed to be caused by the plasma interactions with the cometary and solar wind plasma. During the SIR events, the solar wind ion velocity distributions have a significant angular broadening affecting the plasma moment calculations. In addition, the increase in energy of the solar wind proton signal shows by the RPC-IES data in panel $\mathrm{e}$ indicates that there was indeed a velocity increase. 


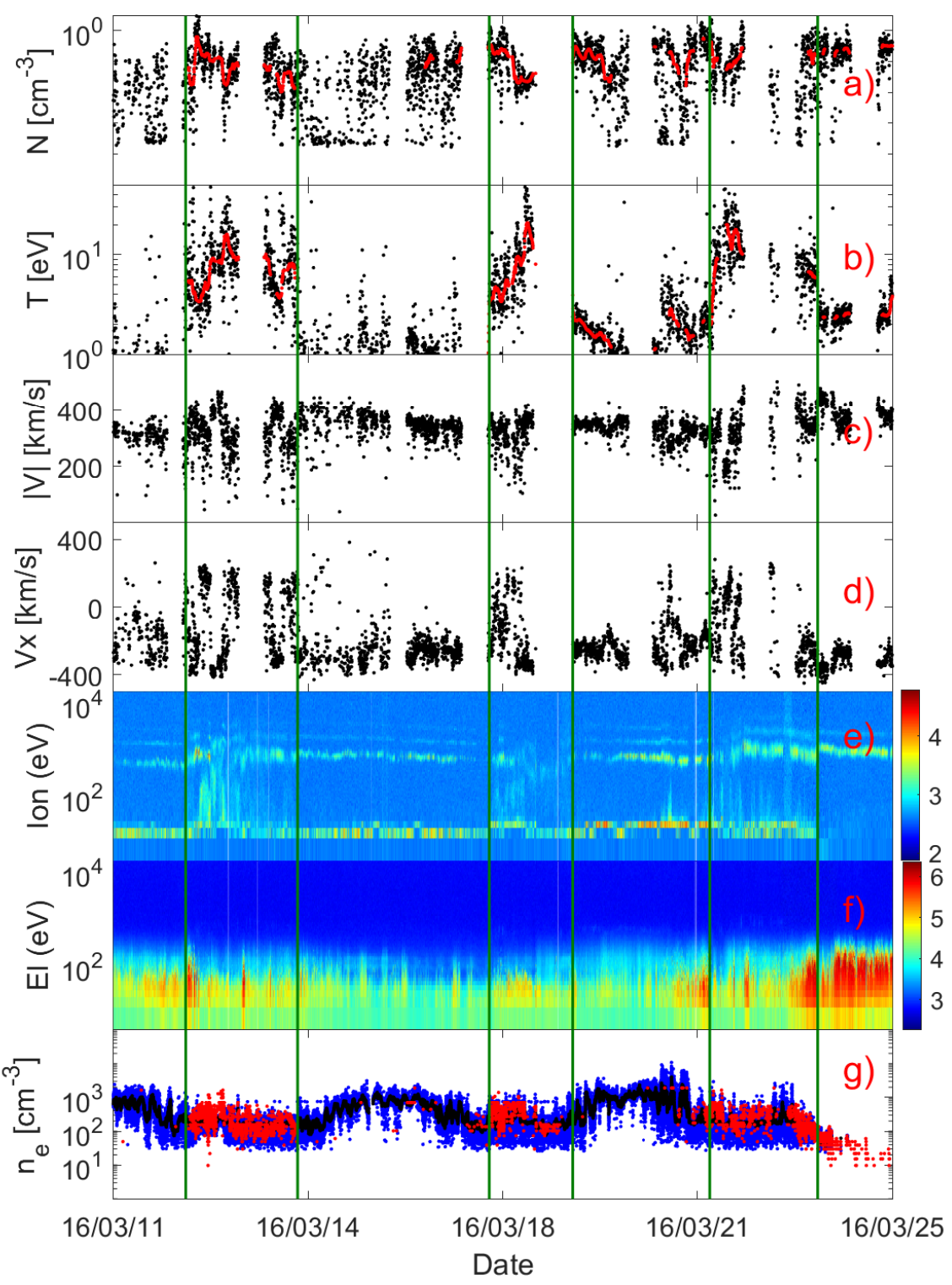

Fig. 10. Three SIR events during March 2016 near comet 67P. The panels show, from top to bottom: solar wind proton density (log-scale), solar wind proton temperature (log-scale), solar wind proton speed, solar wind velocity component parallel to the Sun-comet line, ion counts (color bar is in log-scale showing the exponents for the counts), electron (El) counts, plasma density from RPC-MIP active (nonsmoothed as blue and moving median as black), and passive (red) modes as a function of time. In panels $a$ and $b$, the black dots show nonsmoothed plasma densities and red points are 50-point moving median. In panel $e$, the signal near $1 \mathrm{keV}$ is caused by solar wind protons while the signal below $100 \mathrm{eV}$ shows the cometary ions. The green vertical lines delimit the SIR intervals. Time axis format is [YY/MM/DD].
Furthermore, the RPC-IES instrument measured increased cometary ion counts and increased suprathermal electron $(>70 \mathrm{eV})$ counts (panel $\mathrm{f}$ ) during the SIR. Both are typical features near the comet during SIR interaction, as reported by Edberg et al. (2016a) and Hajra et al. (2018).

We note that the Rosetta orbiter spacecraft made a night-side excursion (Behar et al. 2018; Volwerk et al. 2018) starting from March 24, 2016, and moved away from the comet surface up to $1000 \mathrm{~km}$. The drop in the total plasma density observed during the third SIR period (March 24-March 25) is associated with this spacecraft maneuver, aiming to move away from the comet, and therefore in a less dense plasma, as the plasma density decreases with cometocentric distance (Edberg et al. 2015; Heritier et al. 2017).

The RPC-MIP electric field measurements at the plasma frequency, characteristic of Langmuir waves, are essentially observed during the SIR intervals (corresponding to the blue points in panel g). Thus, it is likely that they are caused by the SIR impact on the comet. The Langmuir wave emissions seem to occur throughout the stream interface as well as during the high-speed stream part. A possible scenario behind the wave emissions during SIRs is described in Sect. 6.

\subsection{Coronal mass ejection impact with comet and Langmuir waves}

In addition to SIRs, we find that CME impacts on comet 67P are responsible for electric field activity at the plasma frequency within the inner cometary plasma environment.

Two CMEs were interacting with the comet in March 2015 when Rosetta was 2.18 AU away from the Sun, as the outgassing of the neutrals was moderate and the induced magnetosphere was not yet fully developed. Strong and long-lasting electric field emissions are observed in the RPC-MIP electric field data (passive spectra) and are expected to be associated to the CME interactions.

Figure 11 shows the passive and active plasma densities extracted from (i) the RPC-MIP electric field spectra (red points) and (ii) the mutual impedance spectra (blue points), respectively, during the $\mathrm{CME}$ interactions. The shock driven by the CME reached the comet on the March 5, 2015 at 21:25 UT, while the trailing edge of the second CME passed the spacecraft around the March 8 at 18:32 UT. The CME sheath is indicated with a green area while both CME ejectas are indicated in gray in Fig. 11. As the shock driven by the CME(s) 


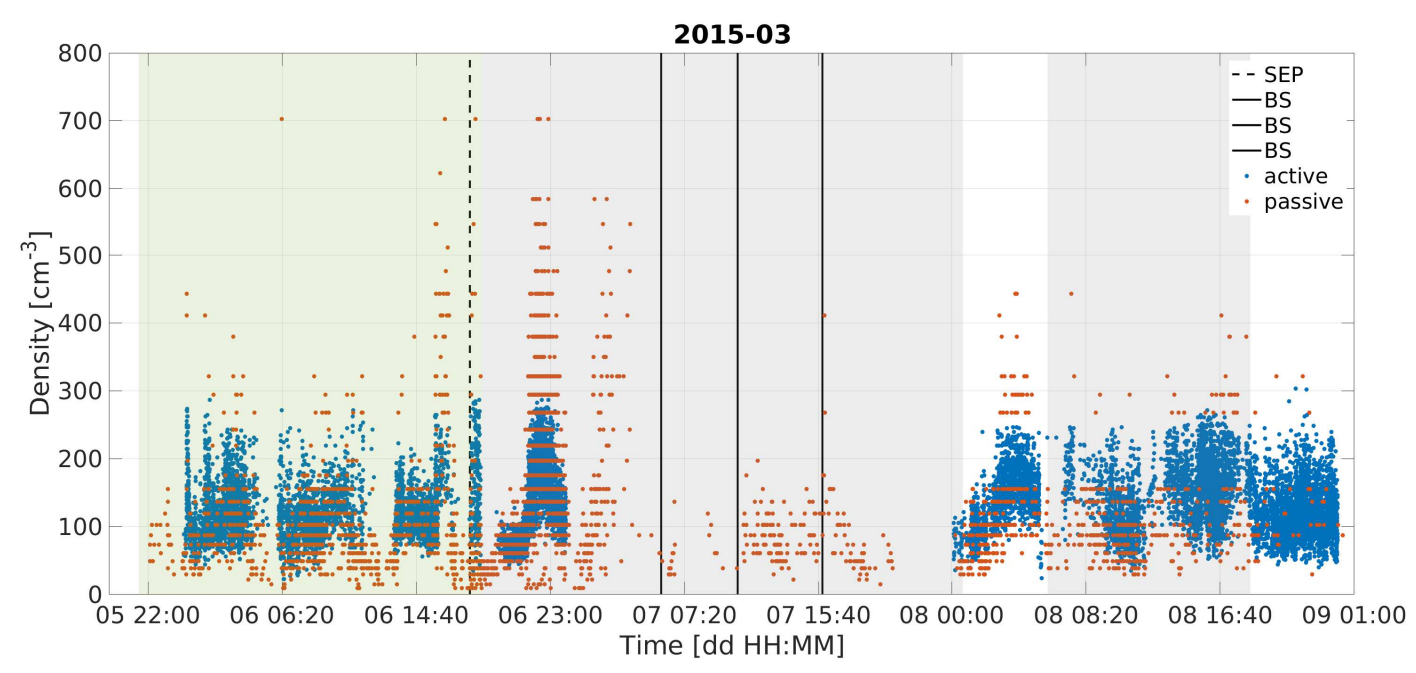

Fig. 11. Cometary plasma density extracted from the RPC-MIP active (blue) and passive (red) modes during two interacting CMEs in March 2015. The CME sheath region is indicated with a green area, while the ejectas are marked in gray. The dashed line shows the beginning of the SEP event, while the infant bow shock locations given by Gunell et al. (2018) are indicated by solid lines.

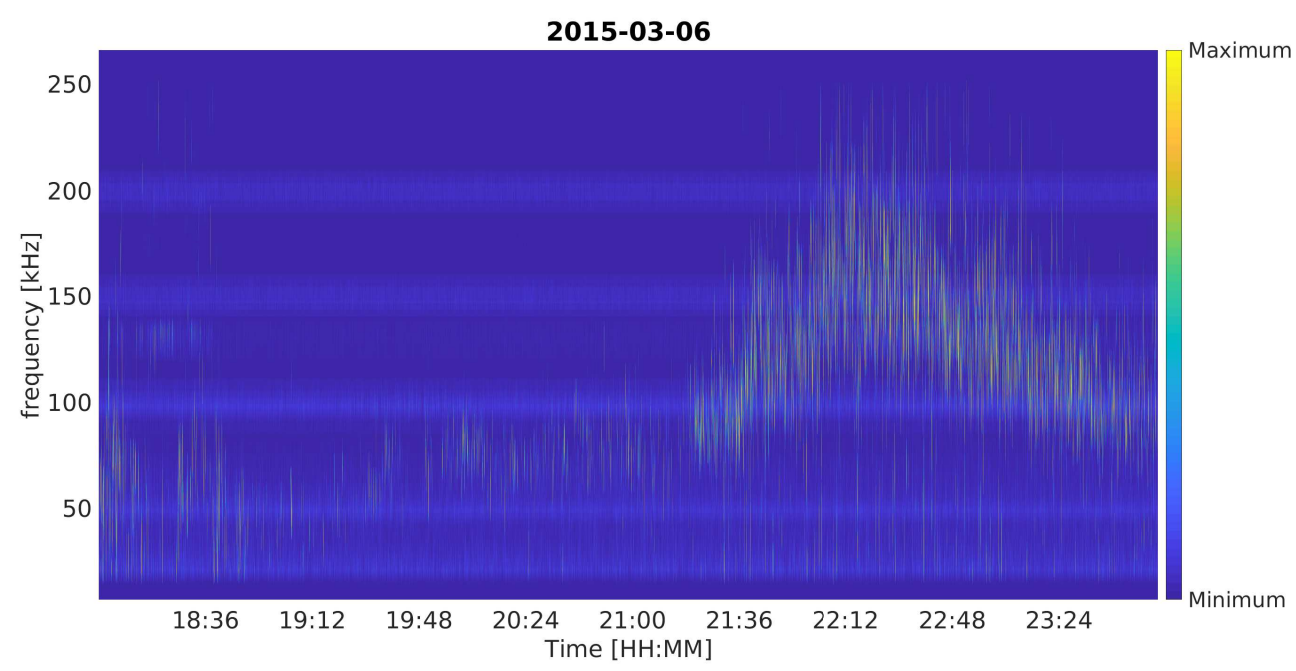

Fig. 12. Example spectrogram showing electric field emissions measured by RPC-MIP passive mode during the SEP and CME events on the March 6,2015 . The spectra have been normalized so that the color scale goes from minimum to maximum power.

reached comet $67 \mathrm{P}$, around $21 \mathrm{UT}$ on the March 5, intense and numerous electric field emissions are observed at the plasma frequency. At 18:00 UT on March 6, 2015 the electric field emissions became extremely intense and the enhanced emissions lasted until midnight (see the spectrogram in Fig. 12). The reason for the increased wave activity might be the solar energetic particle (SEP) event that was observed at the comet starting at the same time as the enhanced emissions (Wellbrock et al. 2018, manuscript in preparation). Solar energetic particles consist of electron beams traveling along magnetic field lines and they are known to excite Langmuir waves in the solar wind. However, Langmuir waves are clearly present already in the CME sheath region. Therefore, it is likely that there is another, non-SEP related phenomenon driving the waves during the sheath. The possible sources of the Langmuir waves are discussed in the following section.

Excluding the first hours of the day, the level of electric field emissions decreased on March 7 compared to the previous day. This time interval roughly corresponds to the ejecta part of the first CME. On March 8, the occurrence rate of the electric field emissions increased once more. That might be due to arrival of the second CME that interacted with the comet during March 8, 2015.

There are times, for example, between 15:00 and 17:00 UT and between 21:00 and 23:00 UT when the passive mode is giving higher estimates for the plasma density than the active mode. The RPC-MIP active mode was operating in LDL mode during the whole day, where density can only be measured up to $300 \mathrm{~cm}^{-3}$, as explained in Sect. 4. Hence, the passive measurements by RPC-MIP complement the measurements made by the RPC-MIP active mode.

\section{Discussion}

As shown in this study, Langmuir waves were present near comet 67P from late 2014 until the end of September 2016 despite the activity phase of the comet. While Rosetta was outside the solar wind cavity, we were able to identify large-scale solar wind structures that were interacting with the comet during the days of numerous Langmuir wave emissions. As shown in Table 1, the strong wave activity was mostly related to SIRs. 
It is not clear what is causing Langmuir waves in the nearcomet environment during interaction with large-scale solar wind structures. In this section, we discuss potential scenarios that could drive Langmuir waves during SIRs or CME sheath regions and also the problems related to them.

Langmuir waves were also present near the perihelion period, when Rosetta was not able to see the solar wind signal. Hence, we have not been able to detect possible solar wind drivers for these wave events. However, in this section, we show that during the last month of the mission, when Rosetta made several close approaches to the cometary surface and there were no solar wind events, the occurrence of Langmuir waves seems to correlate with cold electron observations. Hence, we discuss a possible relationship between Langmuir waves and cold electrons that could play a role in the numerous wave emissions near perihelion.

\subsection{Langmuir wave emissions at comet 67P during SIR impact}

First, we present different mechanisms that might explain the Langmuir wave observations during SIRs based on the fact that Langmuir waves can be expected in the presence of an electron beam moving parallel to the magnetic field. The first scenario is based on the electrons that are accelerated at the SIRdriven shocks. The SIR regions are created when a fast solar wind flow overtakes the slow wind ahead of it. A compression region is formed within the stream interaction region. At large heliocentric distances ( $>2 \mathrm{AU}$ ) the SIR regions are typically confined between forward and reverse shocks (Gosling et al. 1976; Hundhausen \& Gosling 1976; Echer et al. 2010) but a forwardreverse shock pair may even be observed at $1 \mathrm{AU}$ in extreme cases (Jian et al. 2006). The forward shock is moving in the direction of the solar wind flow, expanding the structure into the slow wind, while the reverse shock is moving against the solar wind flow and also spreading the structure. Thus, the structure becomes wider with increasing heliocentric distance.

Solar wind interaction region-related shocks are known to heat and accelerate the solar wind electrons, creating enhanced suprathermal electron fluxes upstream of the forward and reverse shocks (>70 eV; Gosling et al. 1993). These suprathermal electrons are created when the shock-heated electrons are leaked into a region upstream from the shock. The leaked electrons are streaming sunward along the magnetic field lines and are thus counterstreaming relative to the solar wind suprathermal strahl component (Stverak et al. 2009; Pierrard et al. 2016). The counterstreaming electron (CSE) beams can lead to Langmuir wave emission due to beam-plasma instabilities. Steinberg et al. (2005) found that CSEs were observed at high-speed streams followed by SIRs at $1 \mathrm{AU}$ even if the SIRs were not bounded by shocks. The counterstreaming periods occurred around two days after the passage of the SIR. At times, CSEs may also be seen within the slow solar wind preceding a SIR. Steinberg et al. (2005) studied electron energies starting from $70 \mathrm{eV}$ up to $\sim 1000 \mathrm{eV}$. Even if usually the shock pair has not yet developed at 1 AU, a certain SIR can be magnetically connected to a SIR at larger heliocentric distances where the shocks are already present. The leaked electrons accelerated by the forward and reverse shocks can travel along the field lines to reach the Earth's orbit. Thus, Steinberg et al. (2005) suggested that the CSEs observed by the authors at $1 \mathrm{AU}$ would originate from SIR-related shocks beyond 2 AU. The presence of CSEs at 1 AU continues as long as there is a magnetic connection to a SIR at large heliocentric distances. A statistical study of CSEs in the vicinity of SIRs using STEREO data was carried out by Lavraud et al. (2010). The authors observed, similarly to Steinberg et al. (2005), that CSE preferentially occurred before and after the stream interface passed by the spacecraft. That was true even if the bounding shocks had not yet developed.

In this paper, we present observations of Langmuir wave emissions during the SIR intervals studied by Hajra et al. (2018) and during three additional SIRs in March 2016. The SIR interval nearest to the Sun occurred at 2.5 AU and the farthermost at 3.8 AU. If the SIRs are not bounded by shocks when reaching the comet, they are still likely connected to shocks at larger heliocentric distances, as in the SIRs studied by Steinberg et al. (2005) at 1 AU. Thus, CSE populations would be expected to occur prior to the stream interface in the low-speed part and in the high-speed stream part.

However, as shown in Figs. 8 and 10, we observe most of the emissions predominantly during the passage of the stream interface. While the CSEs observed by Steinberg et al. (2005) could explain Langmuir wave emissions during the high-speed stream parts, the source for Langmuir waves at the stream interface remains unknown.

The origin of suprathermal electrons near the comet is still unclear. It has been suggested in the literature that solar wind electrons are accelerated towards the comet parallel to the magnetic field by an ambipolar electric field caused by the pressure gradient (Madanian et al. 2016a; Deca et al. 2017). This would lead to electron heating in parallel to the magnetic field. Because the solar wind density at heliocentric distances greater than $2 \mathrm{AU}$ is less than $1.64 \mathrm{~cm}^{-3}$ (Pierrard et al. 2016), the solar wind density cannot fully explain the suprathermal electron density near the comet that varies from $10^{2}$ to $10^{1} \mathrm{~cm}^{-3}$ (Myllys et al. 2019) from 2 to 3.5 AU. However, the accelerated solar wind electrons can explain part of the observed suprathermal electron velocity distribution, as suggested by Myllys et al. (2019). To excite Langmuir waves, the solar wind electrons accelerated by the ambipolar electric field should form a bump in the local electron velocity distribution. During non-SIR or CME intervals this does not seem to be the case and there is no guarantee that the bump is formed even during the SIR or CME sheath intervals, when the solar wind electron velocity distribution is heated and the density is higher.

The electron beam causing the Langmuir waves can also be generated locally. In that case, we would expect to see some local heating of electrons in the cometary environment that would lead to the creation of the electron beam or bump in the velocity distribution. As seen in Fig. 10 and reported by Edberg et al. (2016a) and Hajra et al. (2018), the SIR intervals show enhanced suprathermal electron fluxes produced by unknown acceleration or heating mechanisms that could be related to the solar wind electron acceleration in the near-comet environment evidenced through numerical simulations of solar wind-comet interactions (Deca et al. 2017).

Based on the results shown in this study, it is not possible to identify the most likely origin of Langmuir waves, and this question remains open, to be solved in future studies.

\subsection{Langmuir wave emissions at comet 67P during CME impact}

The most likely mechanism causing the wave activity during CME passages is related to the electrons accelerated by the CME-driven shock. The Langmuir waves caused by the shock-accelerated electrons are typically observed upstream of the shock (Thejappa \& MacDowall 2000) and a wave mode 


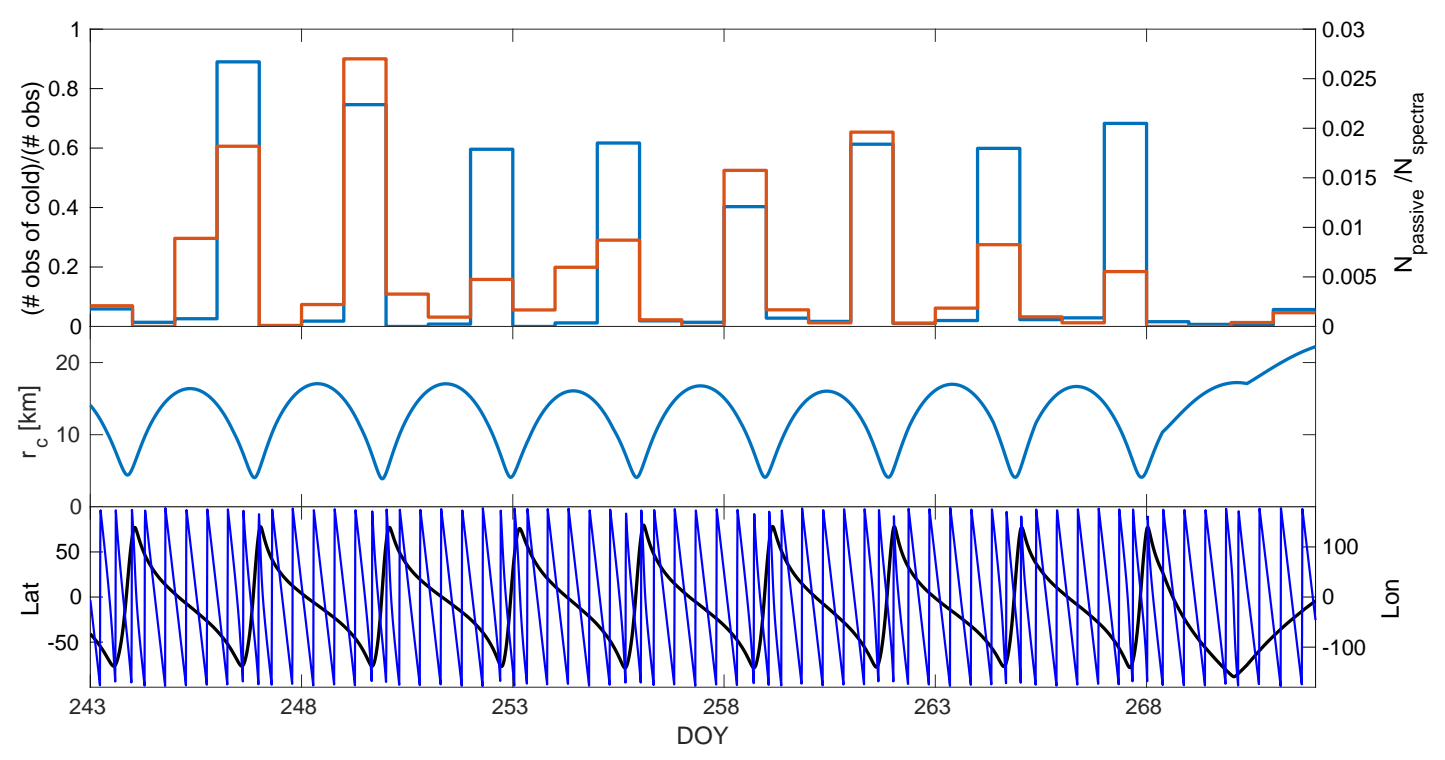

Fig. 13. Upper panel: daily ratio of the number of RPC-MIP active spectra with cold electrons to all measured RPC-MIP active spectra (blue bars, left $y$-axis) and ratio of the number of RPC-MIP passive spectra with electric field emissions to all measured RPC-MIP passive spectra (red bars, right $y$-axis). The horizontal axis indicates the day of the year in 2016. Middle panel: cometocentric distance of Rosetta in km. Bottom panel: latitude (black line, left $y$-axis) and longitude (blue line, right $y$-axis) of Rosetta.

conversion of Langmuir waves leads to type II radio emissions (Bale et al. 1999). However, during the CME event shown in Sect. 5.2, the majority of the Langmuir wave emissions were observed within the sheath region (i.e., downstream of the shock).

Planetary bow shocks, like the one in front of the Earth's magnetosphere, can accelerate electrons. Part of the electrons can leak back to the upstream solar wind and propagate along the magnetic field lines. The leaked foreshock electrons are expected to excite Langmuir waves via the bump-on-tail instability. Several authors have reported observations of the Langmuir waves related to planetary bow shocks (Crawford et al. 1990; Scarf et al. 1971; Lacombe et al. 1985). A bow shock is also assumed to form in front of a comet and be the first boundary separating the solar wind from the cometary plasma. The Rosetta spacecraft never encountered the bow shock because it was operated at short distances from the comet nucleus, much shorter than the actual bow shock distance. During the day-side excursion (Edberg et al. 2016b) Rosetta did not leave the solar wind ion cavity. The time period before and after Rosetta entered the solar wind ion cavity was mostly characterized by very strongly deflected solar wind with only modest heating and little slowing down (Behar et al. 2017; Nilsson et al. 2017a). However, Gunell et al. (2018) showed evidence of an infant bow shock before and after perihelion at large heliocentric distances (2.2 and 2.4 AU). The infant bow shock is defined as an early-stage structure of what is assumed to later evolve into a fully developed cometary bow shock. Three infant bow shock observations were made on March 7, 2015, that is during the CME event shown in Fig. 11. Gunell et al. (2018) reported electron heating near the infant bow shock, but it is not known if the electrons can be accelerated by the infant bow shock. According to Fig. 11, the infant bow shock and the enhanced emissions of Langmuir waves are not concurrent, meaning that there is hardly any relationship between them.

\subsection{Langmuir wave observations near the comet surface and cold electrons}

During the last month of the mission in September 2016, Rosetta did several flybys of the comet and the RPC-MIP active mode A73, page 14 of 16 observed cold electrons near the surface. In addition, the RPCMIP passive mode detected electric field emissions at plasma frequency during the Rosetta flybys.

The daily ratio of cold electrons to all measured RPC-MIP active spectra during September 2016 is shown in Fig. 13. The cold electron observations are compared with the occurrence rate of electric field emissions in the passive spectra. With the exception of the beginning of the month, when a SIR event (Hajra et al. 2018) was observed between the September 1 and 3, there were no other notable solar wind events observed at the comet. Hence, the time interval is ideal for studying the relationship between cold electrons and Langmuir wave emissions. The lower panel of Fig. 13 shows the cometocentric distance of Rosetta as well as its latitude and longitude.

The highest occurrence rate of cold electrons is observed together with enhanced Langmuir wave activity, both predominantly at low altitudes and, at least in September 2016, in the southern hemisphere of the comet (not shown here).

During the last four months of the mission, the outgassing rate was seen to be higher in the southern hemisphere compared to the northern hemisphere (Heritier et al. 2018), and the mean plasma density was also higher at southern latitudes (Hajra et al. 2018). However, it is unclear why the cold population exists at such large heliocentric distances where the electron-neutral collision rate should be very low. Nonetheless, as the Langmuir waves and the cold electron locations observed by RPC-MIP, as well as their occurrence rates, seem to correlate well, we think there might be a relationship between cold electrons and Langmuir waves. It is possible that the waves are excited near the comet surface by some local process related to the cold electrons.

Because the cold electron density peaked near perihelion (Engelhardt et al. 2018), it is likely that the strong and frequent Langmuir wave activity inside the solar wind cavity from June to August 2015 is also partly related to cold electrons. In any case, understanding the mechanism responsible for Langmuir wave excitation in the presence of cold electrons is beyond the scope of this study, and will be addressed in future studies. 


\section{Conclusions}

We studied electric field emissions at the plasma frequency near comet 67P observed by the RPC-MIP instrument on board the Rosetta spacecraft. While operating in passive mode, RPC-MIP can be used as a natural wave plasma analyzer.

The electric field emissions were observed in around $1 \%$ of all the measured RPC-MIP passive spectra. The emission was observed sporadically throughout the time interval when Rosetta was orbiting the comet. The days of highest percentage of emission compared to all measured RPC-MIP passive spectra were found to occur either near the perihelion or when the comet was interacting with large-scale solar wind structures, like SIRs or CMEs. We discuss different scenarios that could cause Langmuir wave emissions during solar wind transient events, but in-situ observations beyond Rosetta are needed in order to distinguish the exact mechanism.

Our analysis reveals new information about the performance of the RPC-MIP active measurements. The accuracy of the RPCMIP mutual impedance measurements used for plasma density estimations depend on the submode of the instrument. It is shown that when the cold electrons component dominates the total electron density, the SDL anti-phase mode can underestimate the plasma density. This further confirms the simulation results by Wattieaux et al. (2019) and Gilet et al. (2020).

Acknowledgements. Rosetta is an ESA mission with contributions from the member states and from NASA. Work at LPC2E is supported by ESEP, CNES and by ANR under the financial agreement ANR-15-CE31-0009-01. We acknowledge use of NASA/GSFC's Space Physics Data Facility's CDAWeb service, ESA's planetary science archive (PSA) and OMNI data. E.P. acknowledges the NASA Living With a Star Jack Eddy Postdoctoral Fellowship Program, administered by UCAR's Cooperative Programs for the Advancement of Earth System Science (CPAESS) under award no. NNX16AK22G. The work of L.T. is supported by the Academy of Finland (grant number 322544). A.W. acknowledges support of STFC consolidated grants ST/N000722/1 and ST/S000240/1.

\section{References}

Bale, S. D., Reiner, M. J., Bougeret, J. L., et al. 1999, Geophys. Res. Lett., 26, 1573

Béghin, C., Hamelin, M., Lebreton, J. P., et al. 2017, J. Geophys. Res.: Space Phys., 122, 6964

Behar, E., Lindkvist, J., Nilsson, H., et al. 2016, A\&A, 596, A42

Behar, E., Nilsson, H., Alho, M., Goetz, C., \& Tsurutani, B. 2017, MNRAS, 469, S396

Behar, E., Nilsson, H., Henri, P., et al. 2018, A\&A, 616, A21

Briand, C., Mangeney, A., \& Califano, F. 2007, Phys. Lett. A, 368, 82

Broiles, T. W., Livadiotis, G., Burch, J. L., et al. 2016a, J. Geophys. Res.: Space Phys., 1217407

Broiles, T. W., Burch, J. L., Chae, K., et al. 2016b, MNRAS, 462, S312

Burch, J. L., Goldstein, R., Cravens, T. E., et al. 2007, Space Sci. Rev., 128, 697

Carr, C., Cupido, E., Lee, C. G. Y., et al. 2007, Space Sci. Rev., 128, 629

Clark, G., Broiles, T. W., Burch, J. L., et al. 2015, A\&A, 583, A24

Crawford, G. K., Strangeway, R. J., \& Russell, C. T. 1990, Geophys. Res. Lett., 17,1805

Deca, J., Divin, A., Henri, P., et al. 2017, Phys. Rev. Lett., 118, 205101

Deng, X. H., Matsumoto, H., Kojima, H., et al. 2004, J. Geophys. Res.: Space Phys., 109 A05206

Echer, E., Tsurutani, B. T., \& Guarnieri, F. L. 2010, Adv. Space Res., 45, 798

Edberg, N. J., Eriksson, A. I., Odelstad, E., et al. 2015, Geophys. Res. Lett., 42, 4263

Edberg, N. J., Eriksson, A. I., Odelstad, E., et al. 2016a, J. Geophys. Res.: Space Phys., 121, 949
Edberg, N. J., Alho, M., André, M., et al. 2016b, MNRAS, 462, S45 Engelhardt, I. A., Eriksson, A. I., Vigren, E., et al. 2018, A\&A, 616, A51

Eriksson, A. I., Boström, R., Gill, R., et al. 2007, Space Sci. Rev., 128, 729

Eriksson, A. I., Engelhardt, I. A., André, M., et al. 2017, A\&A, 605, A15

Galand, M., Héritier, K. L., Odelstad, E., et al. 2016, MNRAS, 462, S331

Gilet, N., Henri, P., Wattieaux, G., Cilibrasi, M., \& Béghin, C. 2017, Radio Sci., 52,1432

Gilet, N., Henri, P., Wattieaux, G., et al. 2020, A\&A, 640, A110

Gosling, J. T., Hundhausen, A. J., \& Bame, S. J. 1976, J. Geophys. Res., 81, 2111

Gosling, J. T., Bame, S. J., Feldman, W. C., et al. 1993, Geophys. Res. Lett., 20, 2335

Grard, R., Pedersen, A., Trotignon, J. G., et al. 1986, Nature, 321, 290

Grasset, O., Dougherty, M. K., Coustenis, A., et al. 2013, Planet. Space Sci., 78, 1

Gunell, H., Goetz, C., Wedlund, C. S., et al. 2018, A\&A, 619, A2

Hajra, R., Henri, P., Vallières, X., et al. 2017, A\&A, 607, A34

Hajra, R., Henri, P., Myllys, M., et al. 2018, MNRAS, 480, 4544

Henri, P., \& RPC-MIP team 2018, User Guide to the RPC-MIP Science Datasets in the ESA's Planetary Science Archive (PSA) - version 1.5, Planetary Science Archive

Henri, P., Briand, C., Mangeney, A., et al. 2009, J. Geophys. Res., 114, A03103

Henri, P., Vallières, X., Hajra, H., et al. 2017, MNRAS, 469, S372

Heritier, K. L., Henri, P., Vallières, X., et al. 2017, MNRAS, 469, S118

Heritier, K. L., Galand, M., Henri, P., et al. 2018, A\&A, 618, A77

Hundhausen, A. J., \& Gosling, J. T. 1976, J. Geophys. Res., 81, 1436

Jian, L., Russell, C. T., Luhmann, J. G., \& Skoug, R. 2006, Solar Phys., 239, 337

Kasaba, Y., Kojima, H., Moncuquet, M., et al. 2020, Space Sci. Rev., 2016, 65

Kennel, C. F., Coroniti, F. V., Scarf, F. L., et al. 1986, Geophys. Res. Lett., 13, 921

Khalilpour, H., \& Foroutan, G. 2012, Astrophys. Space Sci., 338, 49

Lacombe, C., Mangeney, A., Harvey, C. C., \& Scudder, J. D. 1985, J. Geophys. Res.: Space Phys., 90, 73

Landau, L. 1946, J. Phys. (USSR), 10, 45

Lavraud, B., Opitz, A., Gosling, J. T., et al. 2010, Ann. Geophys., 28, 233

Lin, N., Kellogg, P. J., Goetz, K. A., Monson, S. J., \& MacDowall, R. J. 1999, AIP Conf. Proc., 471, 673

Madanian, H., Cravens, T. E., Rahmati, A., et al. 2016, J. Geophys. Res.: Space Phys., 1215815

McFadden, J. P., Carlson, C. W., \& Boehm, M. H. 1986, J. Geophys. Res.: Space Phys, , 91, 12079

Milillo, A., Fujimoto, M., Murakami, G., et al. 2020, Space Sci. Rev., 216, 93

Myllys, M., Henri, P., Galand, M., et al. 2019, A\&A, 630, A42

Nilsson, H., Lundin, R., Lundin, K., et al. 2007, Space Sci. Rev., 128, 671

Nilsson, H., Wieser, G. S., Behar, E., et al. 2015, A\&A, 583, A20

Nilsson, H., Wieser, G. S., Behar, E., et al. 2017, MNRAS, 469, S252

Pierrard, V., Lazar, M., Poedts, S., et al. 2016, Solar Phys., 291, 2165

Pulupa, M. P., Bale, S. D., \& Kasper, J. C. 2010, J. Geophys. Res.: Space Phys., 115, A0410

Rose, D. V., Guillory, J., \& Beall, J. H. 2005, Phys. Plasmas, 12, 014501

Rouillard, A. P., Lavraud, B., Genot, V., et al. 2017, Planet. Space Sci., 147, 61

Scarf, F. L. 1971, J. Geophys. Res., 76, 7769

Skoug, R. M., Feldman, W. C., Gosling, J. T., McComas, D. J., \& Smith, C. W. 2000, J. Geophys. Res.: Space Phys., 105, 23069

Steinberg, J. T., Gosling, J. T., Skoug, R. M., \& Wiens, R. C. 2005, J. Geophys. Res.: Space Phys., 110, A06103

Štverák, Š., Maksimovic, M., Trávníček, P. M., et al. 2009, J. Geophys. Res.: Space Phys., 114, A05104

Thejappa, G., \& MacDowall, R. J. 2000, ApJ, 544, L163

Thorne, R. M., \& Summers, D. 1991, Phys. Fluids B: Plasma Phys., 3, 2117

Treumann, R. A. 2009, A\&ARv, 17, 409

Trotignon, J. G., Béghin, C., Lagoutte, D., et al. 2006, Adv. Space Res., 38, 686

Trotignon, J. G., Michau, J. L., Lagoutte, D., et al. 2007, Space Sci. Rev., 128, 713

Volwerk, M., Goetz, C., Richter, I., et al. 2018, A\&A, 614, A10

Wattieaux, G., Gilet, N., Henri, P., Vallières, X., \& Bucciantini, L. 2019, A\&A, 630, A41

Wellbrock, A., Jones, G., Coates, A., et al. 2018, Eur. Planet. Sci. Congr., EPSC2018-964 


\section{Appendix A: SIR observations at $1 \mathrm{AU}$}

Section 5.1 of the main article shows three SIR events that interacted with comet 67P causing an enhanced Langmuir wave activity. As the comet is lacking an upstream solar wind monitor and SIRs cannot be identified from Rosetta data in a straightforward manner, as they can from data from the spacecraft at $1 \mathrm{AU}$, we show the appearance of the SIRs at 1 AU in Fig. A.1. We estimated arrival times for the SIR structures shown in Fig. A.1 to comet 67P using the CDPP Propagation Tool ${ }^{1}$ (Rouillard et al. 2017) to check that they match with the SIR intervals indicated in Fig. 10 of the main article.

Figure A.1 shows the amplitude of interplanetary magnetic field as well as solar wind speed, density, temperature, and pressure at $1 \mathrm{AU}$. The data were extracted from the NASA/GSFC OMNI data set through OMNIWeb. The first SIR occurred between March 6 and March 7, the second during March 11 and the third between March 14 and March 15 at 1 AU (Fig. A.1). Correspondingly, the structures reached Rosetta around March 12, 17, and 20 (Fig. 10).

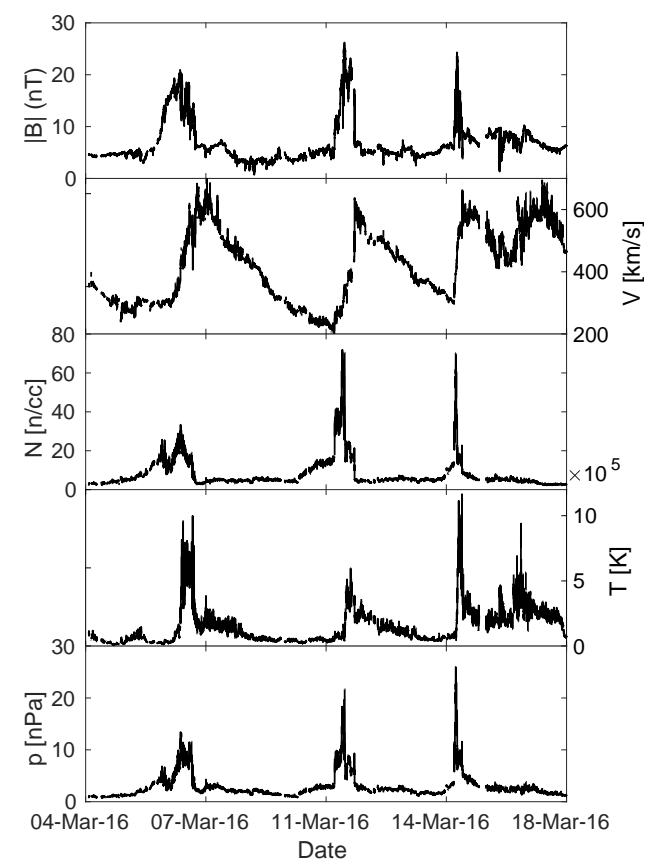

Fig. A.1. Solar wind conditions during three SIR events at 1 AU. The panels show, from top to bottom, the magnitude of the interplanetary magnetic field (nT), solar wind speed $\left(\mathrm{km} \mathrm{s}^{-1}\right)$, solar wind density $\left(\mathrm{n} \mathrm{cc}^{-1}\right)$, solar wind temperature $(\mathrm{K})$, and solar wind pressure $[\mathrm{nPa}]$. 